\title{
Dense gas and cold dust in the dark core B217
}

\author{
S. Hotzel ${ }^{1}$, J. Harju ${ }^{2}$, D. Lemke ${ }^{1}$, K. Mattila ${ }^{2}$, and C. M. Walmsley ${ }^{3}$ \\ 1 Max-Planck-Institut für Astronomie, Königstuhl 17, 69117 Heidelberg, Germany \\ 2 Observatory, PO Box 14, 00014 University of Helsinki, Finland \\ 3 Osservatorio Astrofisico di Arcetri, Largo E. Fermi 5, 50125 Firenze, Italy
}

Received 11 October 2000 / Accepted 28 March 2001

\begin{abstract}
The Barnard object B217 was observed in the infrared and radio region. The $170 \mu \mathrm{m}$ continuum was detected with ISOPHOT, the ammonia $1.3 \mathrm{~cm}$ radio lines with the Effelsberg $100 \mathrm{~m}$-telescope. Mapping B217SW in $(J, K)=(1,1)$ and $(2,2)$ inversion lines revealed the temperature and density distribution of the gas and made it possible to investigate the dynamical state of this dense core inside B217. The ISOPHOT Serendipity Survey (ISOSS) detected the cold dust emission of B217 in all of 3 slews crossing the region. Combining ISOSS with IRAS data, we derive the core parameters of the dust from FIR emission and compare them with the $\mathrm{NH}_{3}$ data, which sample the densest region of the core. This study shows the power of combining ISOSS $170 \mu \mathrm{m}$ with IRAS/HIRES data in order to study the dust characteristics in nearby star forming regions on small spatial scales. The $(170 \mu \mathrm{m} / 100 \mu \mathrm{m})$ dust colour temperature is $11 \mathrm{~K}-12 \mathrm{~K}$ in the dense cores and $12 \mathrm{~K}-14 \mathrm{~K}$ in the other regions of B217. The low dust temperatures cannot be explained by attenuation of the interstellar radiation field alone and may reflect a change in the optical properties of the dust as compared to diffuse clouds. In B217SW, molecular depletion through freeze-out onto grains is suggested by the comparison of our FIR and $\mathrm{NH}_{3}$ data with previous $\mathrm{C}^{18} \mathrm{O}$ observations. On the basis of our ammonia data investigation, we find in B217SW dense gas with kinetic temperatures between $9 \mathrm{~K}$ and $12 \mathrm{~K}$, increasing outwards. Using near-infrared extinction and $\mathrm{NH}_{3}$ collisional excitation calculations, the fractional ammonia abundance $\left(N\left(\mathrm{NH}_{3}\right) / N\left(\mathrm{H}_{2}\right)\right)$ is found to be $3-5 \times 10^{-8}$, and the comparison of gas and dust observations supports this range. Knowing the ammonia abundance, we calculate the thermal, turbulent and gravitational energies of the dense core, which appears to be close to hydrostatic equilibrium. Our results are compatible with B217SW being now on the verge of collapse or in an early collapse phase.
\end{abstract}

Key words. ISM: clouds - ISM: individual objects: Barnard 217 - ISM: molecules - infrared: ISM - radio lines: ISM - surveys

\section{Introduction}

High-mass stars form in clusters in large molecular clouds. Isolated star formation takes place in small dense cores. These may or may not be embedded in larger clouds; their evolution can be regarded as independent of physical conditions in the rest of the cloud, and single star forming events can be studied. The stars produced in this scenario are always low-mass stars. As the number of known sites of isolated star formation is limited, while many theoretical models have been developed for this star formation mode,

Send offprint requests to: S. Hotzel,

e-mail: hotzel@mpia-hd.mpg.de

* Based on observations with ISO, an ESA project with instruments funded by ESA Member States (especially the PI countries: France, Germany, The Netherlands and the UK) and with the participation of ISAS and NASA. Members of the Consortium on the ISOPHOT Serendipity Survey (CISS) are MPIA Heidelberg, ESA ISO SOC Villafranca, AIP Potsdam, IPAC Pasadena, Imperial College London. it is important to investigate the known cores individually. The study of core properties is an essential ingredient of any further investigation of star formation conditions.

B217 is a dark core near the southeastern end of a large dark filament belonging to the so-called Barnard's Cloud in the central part of the Taurus molecular cloud complex (see the sketch in Gaida et al. 1984). The distance to this nearby star-forming region is $140 \mathrm{pc}$ (Elias 1978). On an optical image, B217 appears as a $15^{\prime} \times 10^{\prime}$ $(0.6 \mathrm{pc} \times 0.4 \mathrm{pc})$ sized dark core, which is well separated from the diffuse sky background (see Fig. 1). The absence of stars on the POSS-II plates (detection limit $22.5 \mathrm{mag}$ ) over several $10 \square^{\prime}$ sized regions across the core implies an extinction of $A_{V}>5 \mathrm{mag}$ relative to the immediate surroundings.

Myers et al. (1983) detected ${ }^{13} \mathrm{CO}$ and $\mathrm{C}^{18} \mathrm{O}$, Myers \& Benson (1983) detected ammonia in B217. The peak position in their $\mathrm{NH}_{3}(J, K)=(1,1)$ inversion line map served as reference position for further studies with other molecules, such as CS (Zhou et al. 1989), HCN, 
HNC (Harju 1989), $\mathrm{HC}_{3} \mathrm{~N}$ (Fuller \& Myers 1993), $\mathrm{C}^{18} \mathrm{O}$, $\mathrm{DCO}^{+} / \mathrm{H}^{13} \mathrm{CO}^{+}$(Butner et al. 1995), CS, $\mathrm{N}_{2} \mathrm{H}^{+}$(Lee \& Myers 1999). All of these are single position observations. Mizuno et al. (1995) and Onishi et al. (1996) mapped $8 \square^{\circ}$ in the Taurus complex in ${ }^{13} \mathrm{CO}$ and $\mathrm{C}^{18} \mathrm{O}$ respectively. Onishi et al. (1998) presented $\mathrm{C}^{18} \mathrm{O}(J=1 \rightarrow 0)$ integrated line intensity maps of $40 \mathrm{C}^{18} \mathrm{O}$ cores identified in the previous survey. Their core No. 22 with 3 roughly equally strong intensity peaks coincides with B217. The nearest $\mathrm{C}^{18} \mathrm{O}$ peak is $\sim 3^{\prime}$ offset relative to the $\mathrm{NH}_{3}$ peak.

In the optical, B217 has two brightness minima which are equally deep. The shapes of the subclumps are similar $\left(\sim 5^{\prime} \times 3^{\prime}\right)$. We denote these clumps as B217SW and B217NE with central (J2000) positions $\alpha=4^{\mathrm{h}} 27^{\mathrm{m}} 47^{\mathrm{s}}, \delta=26^{\circ} 18^{\prime}$ and $\alpha=4^{\mathrm{h}} 28^{\mathrm{m}} 10^{\mathrm{s}}, \delta=26^{\circ} 21^{\prime}$. The labelling follows Goodman et al. (1993), who investigated velocity gradients of dense cores. Reinvestigating the Myers \& Benson (1983) data, they found the velocity gradients in the two parts of the ammonia map to point in almost opposite directions. Even though their $(J, K)=(1,1)$ antenna temperature map does not cover the north-eastern core completely, it shows the double core morphology. In contrast to the optical, the radio emission of $\mathrm{B} 217 \mathrm{NE}$ is weaker and less confined than that of B217SW.

There is a Class-I (Lada \& Wilking 1984; Lada 1987) young stellar object (YSO) in between the cores (IRAS 04248+2612, white triangle in Fig. 1), detected in all but the $12 \mu \mathrm{m}$ IRAS band, with a spectrum monotonically rising towards $100 \mu \mathrm{m}$ and a bolometric luminosity of $0.3 L_{\odot}$ (Chen et al. 1995). At $2 \mu \mathrm{m}$, a cometary shaped nebulosity extends from the YSO $40^{\prime \prime}(5000 \mathrm{AU})$ to the northwest (Tamura et al. 1991), perpendicular to the axis connecting B217NE and B217SW. Hence recent star formation in B217 coexists with two dense cores without detected YSOs. The star(s) formed so far are probably too weak to have a major impact on the dense cores and one suspects that the two dense cores will form low-mass stars themselves. Hence, B217 is of interest as a typical core forming low mass stars and B217SW is a good candidate for being a dense core just prior to star formation.

We have probed the gas in the putatively densest region - B217SW - by ammonia mapping in $(J, K)=(1,1)$ and $(2,2)$ inversion line transitions. This allows us to derive the kinetic temperature $T_{\text {kin }}$, ammonia column density $N\left(\mathrm{NH}_{3}\right)$ and total number density $n$ of the gas. The overall dynamical state of the core is determined by comparing kinetic and gravitational energies.

Far-infrared (FIR) emission of the dust in dense cores typically peaks beyond the $100 \mu \mathrm{m}$ IRAS band. With ISO, extending the accessible FIR wavelength range to $240 \mu \mathrm{m}$, it is now possible to sample the bulk of the emitted energy. We use the ISOPHOT Serendipity Survey (ISOSS) at $170 \mu \mathrm{m}$ and compare it with $100 \mu \mathrm{m}$ and $60 \mu \mathrm{m}$ IRAS/HIRES maps. We derive the dust temperature $T_{\text {dust }}$ and the total mass $M_{\text {dust }}$ of B217 and its dense cores. We emphasize that our FIR and Radio observations do not sample the same spatial volume. However, combining the results from FIR and radio observations, we get a comprehensive picture of star formation conditions in B217.

\section{Observations and data analysis}

\subsection{FIR data}

\subsubsection{ISOSS}

The ISOPHOT Serendipity Survey (ISOSS) (Lemke et al. 1996) recorded the $170 \mu \mathrm{m}$ sky brightness when the satellite was slewing between two pointed observations (details in Bogun et al. 1996). The width of the slews is $\approx 3^{\prime}$ resulting in a total ISOSS sky coverage of $15 \%$. The effective angular resolution of ISOSS (FWHM of a point source profile) is larger than the diffraction limit of $93^{\prime \prime}$ (full width at half maximum (FWHM) of the theoretical footprint model) due to effects of the fast detector movement of up to $8^{\prime} / \mathrm{s}$. Studying the profiles of 6 repeatedly crossed calibration sources, the effective resolution was found to be $2.19^{\prime}$ with a sample standard deviation of $0.08^{\prime}$ (Hotzel 2001). The slew-width is too small to independently determine an effective cross-scan beam profile. Assuming a circular ISOSS beam profile is however a reasonable approximation, as is demonstrated by Stickel et al. (2000). A measurement of the on-board Fine Calibration Source preceded most of the slews of the survey and all slews crossing B217. For conversion of raw detector voltage to surface brightness and removal of cosmic ray hits, the methods of Stickel et al. (2000, see their Table 1, steps 1. and 3.) were used. In contrast to their processing steps necessary for the extraction of point sources, no background was subtracted. Their routine to estimate the background was used instead to flatfield the detector. In order not to affect local changes in the gradient of the background, only one average value (per pixel) for the slew was applied. No flatfield between the slews was performed.

The accuracy of the ISOSS calibration has been checked in Appendix A.1. Summarizing, we regard a 30\% photometric accuracy of ISOSS (in Taurus) as a conservative upper limit.

\subsubsection{IRAS/HIRES}

In order to investigate the FIR energy distribution of the dust and to derive colour temperatures, we complement ISOSS with IRAS $100 \mu \mathrm{m}$ and $60 \mu \mathrm{m}$ intensities. To take advantage of the full angular resolution we use HIRES processed IRAS data (Aumann et al. 1990), for which we chose default HIRES processing except for switching off baseline subtraction. The 20 iterations provided us with maps of $109^{\prime \prime} \times 100^{\prime \prime}$ and $77^{\prime \prime} \times 50^{\prime \prime}$ resolution at $100 \mu \mathrm{m}$ and $60 \mu \mathrm{m}$ respectively (taken from the table of effective beam sizes in the beam sample image at the site of IRAS 04248+2612). These original HIRES maps have been smoothed with non-circular 2-dim. Gauss functions of the required widths and position angles to achieve a resolution of $2.2^{\prime}$ for all FIR data. 


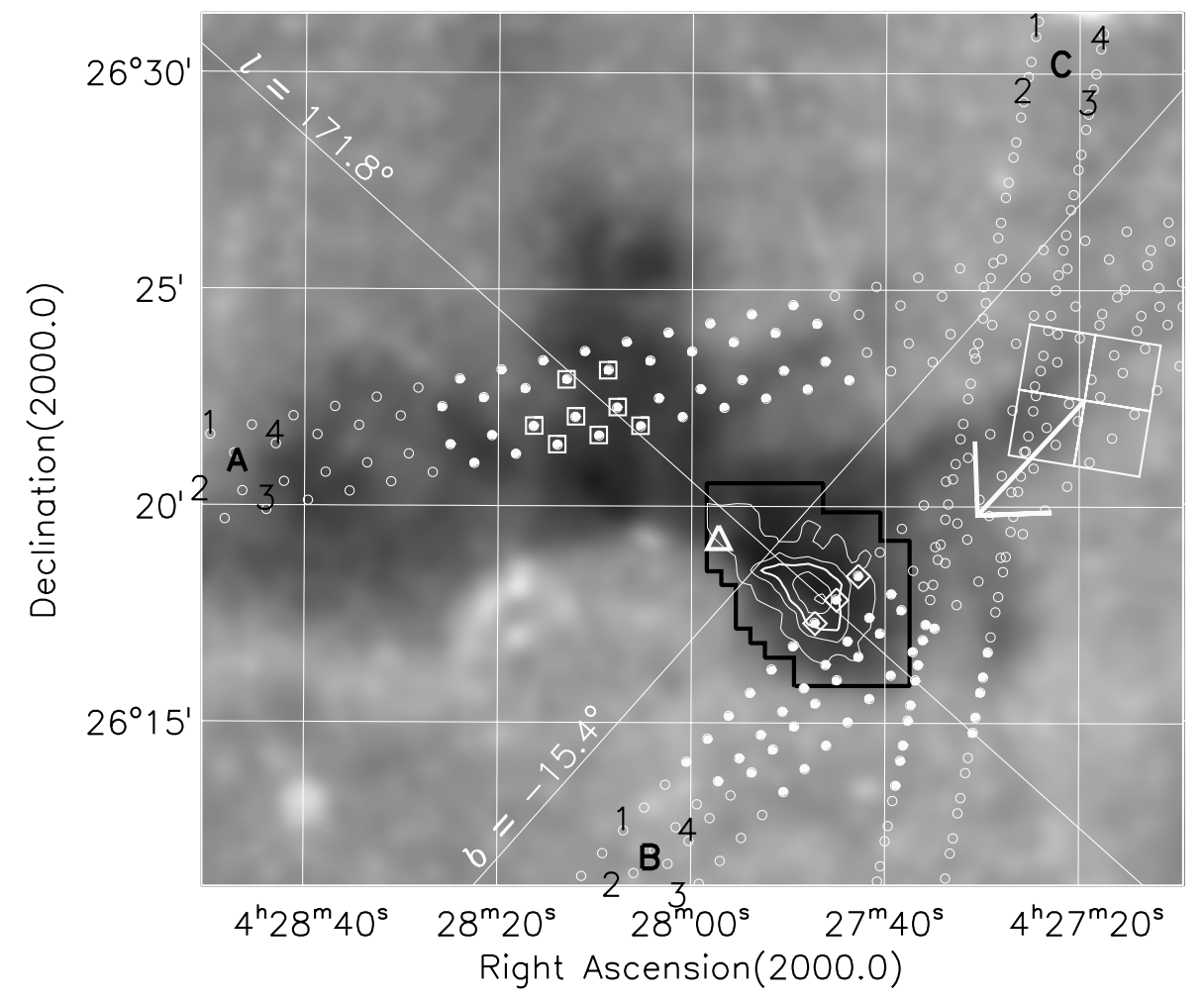

Fig. 1. B217 as dark patch on the POSS-II blue plate. After point sources had been removed, the image was smoothed to $30^{\prime \prime}$. The YSO IRAS 04248+2612 in between B217SW and B217NE is marked by a triangle. $\mathrm{NH}_{3}$ column density contours from our Effelsberg observations of B217SW are overlayed. Figure 3 shows the same contours enlarged. Three ISOSS slews have crossed the dark core; small white circles indicate the individual pixel read-out positions. The field of view of each pixel is $90^{\prime \prime} \times 90^{\prime \prime}$. For easier reference in this paper, we name the slews Slew A (running west to east), Slew B (west to south) and Slew C (south to north). Individual pixels are numbered. Filled circles indicate read-outs, which have been used for ananlysis of the dense cores (Fig. 8). White boxes mark positions of "anomalous" data points (Fig. 6). The detector and pixel apertures are marked on Slew B, Pixel 1\&4 of which have been used in Fig. 9 .
Summarizing Appendix A.2, we regard the photometric accuracy of the $100 \mu \mathrm{m}$ HIRES map to be $30 \%$, limited for small angular scales close to IRAS 04248+2612 by a possible ringing artefact and for the overall emission of B217 by the unknown calibration correction factor. The photometric accuracy of the $60 \mu \mathrm{m}$ HIRES map is similar for the large scale emission. The ringing artefacts affecting investigations of the dense cores close to IRAS $04248+2612$ are however much more serious, because the point source flux density is a factor 2 lower than at $100 \mu \mathrm{m}$, while the expected surface brightness of the cores at least a factor 10 . Hence the $60 \mu \mathrm{m}$ map is used only for a qualitative discussion regarding the FIR emission of dust in B217, but not for a quantitative estimate of temperatures (see Fig. 7).

\subsubsection{Deriving dust colour temperatures}

In order to derive colour temperatures and column densities of the dust, we have read out $I_{100}$ and $I_{60}$ intensities from the HIRES maps at all positions of the ISOSS slews in B 217 (marked in Fig. 1). The zodiacal light contribution to $I_{170}$ has been subtracted individually from the scans, using the DIRBE model. (The offset compensation destriper in the HIRES processing has leveled different zodiacal light contributions to the individual HCONs and an additive offset does not affect our analysis.) Assuming a modified black body radiation with $\nu^{2}$ emissivity,

$I_{\nu} \propto \nu^{2} B_{\nu}\left(T_{\text {dust }}\right)$

we can determine the dust temperature from the slope of a straight line fit to the $\left(I_{100}, I_{170}\right)$ data points, applying the necessary colour corrections (see also Appendix A.1.3) for the specific ISOPHOT $170 \mu \mathrm{m}$ and IRAS $100 \mu \mathrm{m}$ bandpasses. The underlying assumption for this procedure is that we are dealing with an optically thin source at $170 \mu \mathrm{m}$ and $100 \mu \mathrm{m}$ with no steep gradient in the background. Using line-fits to samples of data points, which we identify with objects on morphological grounds, we considerably decrease the scatter inherent to our FIR maps and we avoid the need to manually subtract the background from our data, which usually introduces a large bias. The accuracy of the derived colour temperature is determined by evaluating Eq. (1) for slopes multiplied by $1+\Delta$ and $1-\Delta$, where $\Delta=\sqrt{0.3^{2}+0.3^{2}+\sigma(\text { slope })^{2}}$ is obtained from the ISOSS and HIRES photometric accuracies and the 1-sigma formal error of the line-fit.

If we know the FIR colour temperature and assume an isothermal cloud, we can determine the FIR opacity from the radiative transfer equation in the optically thin limit:

$I_{\nu}=\tau(\nu) B_{\nu}\left(T_{\text {dust }}\right)$

In the following, the quoted values for $I_{\nu}$ are not colour corrected in order to be consistent with the figures. The correction factor $\left(\approx 0.92\right.$ for $I_{170}$ and the SEDs of this paper) has been applied when converting to the physical parameters, i.e. column density and mass.

\subsection{Ammonia observations}

The ammonia observations were carried out in July 1988 with the Effelsberg 100 m-telescope ( $40^{\prime \prime}$ beam at $23 \mathrm{GHz}$ ). We used a $K$-band maser receiver with a typical system 
temperature of about $100 \mathrm{~K}$ on blank sky. The spectrometer used was a 1024 channel autocorrelator split into two halves with a band-width of $6.25 \mathrm{MHz}$ each, centered on the frequencies of the $(J, K)=(1,1)$ and $(2,2)$ inversion transitions of $\mathrm{NH}_{3}$. The velocity resolution obtained was $0.15 \mathrm{~km} \mathrm{~s}^{-1}$. The observations were made using position switching. The map spacing was $20^{\prime \prime}$ around the core centre and $40^{\prime \prime}$ on the outskirts of the map, the extent of which is marked in Fig. 1. The pointing was checked and the spectra were calibrated by using continuum scans towards 3C 123 for which we assumed a main-beam brightness temperature of $4.0 \mathrm{~K}$. This corresponds to an observed flux density of $2.85 \mathrm{Jy}$ (Ott et al. 1994), which takes into account the spatial extension $\left(23^{\prime \prime} \times 5^{\prime \prime}\right)$ of the calibration source. The pointing accuracy was found to be better than $5^{\prime \prime}$.

The hyperfine spectra were reduced using a method described by Harju et al. (1993); $\mathrm{NH}_{3}$ fundamentals can be found in the review by Ho \& Townes (1983). In a $2^{\prime} \times 1.5^{\prime}$ region around the peak position (see Fig. 3), the signal-to-noise ratio (SNR) in the $(1,1)$ line was high enough to determine its excitation temperature and opacity, and the SNR in the $(2,2)$ line was high enough to calculate the partition function. The latter is calculated assuming that only metastable $(J=K)$ rotational levels are populated and the rotational temperature $T_{12}$ (defined by $\left.n(2,2) / n(1,1)=5 / 3 \exp \left(-\Delta E_{12} / k T_{12}\right)\right)$ is characteristic for all metastable levels and the ground state. At positions where the optical depth could not be derived from the hyperfine spectra analysis, optically thin emission in the hyperfine groups and an excitation temperature $T_{\mathrm{ex}}$ (describing the relative population of the upper $(1,1)$ state) of $10 \mathrm{~K}$ were assumed. $T_{12}=10 \mathrm{~K}$ was assumed at positions without a detected $(2,2)$ transition. Using these assumptions we could trace the ammonia column density $N\left(\mathrm{NH}_{3}\right)$ over almost 2 orders of magnitude. The determination of the kinetic temperature $T_{\text {kin }}$ was restricted to the central region, where $T_{\mathrm{ex}}$ and $T_{12}$ could be derived from the measurements. See Harju et al. (1993) for all equations used in this method.

Figure 2 shows the $(J, K)=(1,1)$ spectrum at the peak position, as well as two theoretical spectra revealing the hyperfine structure of the transition.

\section{Results}

\subsection{Gas}

The results of our ammonia observations are presented in Fig. 3, where contours of $\mathrm{NH}_{3}$ column density are overlaid on an image of kinetic temperature. $T_{\text {kin }}$ could be derived only in the central part of the core (converted from $T_{12}$ using the 3-rotational-levels-model of Walmsley \& Ungerechts 1983, with collisional rate coefficients from Danby et al. 1988). In all positions where $T_{\mathrm{ex}}$ and $T_{12}$ could be derived from the measurements we have compared $N\left(\mathrm{NH}_{3}\right)$ values with column densities assuming $T_{\mathrm{ex}}=T_{12}=10 \mathrm{~K}$ (this assumption has to be used in

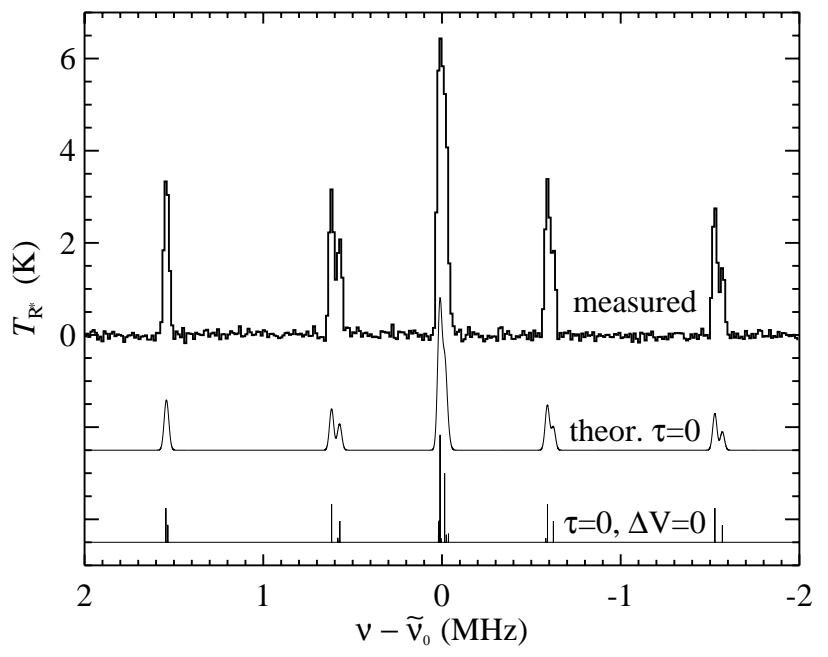

Fig. 2. Hyperfine structure of the $(J, K)=(1,1)$ inversion transition of ammonia. The upper line is the calibrated spectrum at the peak position. The $y$-axis gives the antenna temperature corrected for atmospheric transmission $T_{\mathrm{R}}^{*}$, the $x$ axis gives the frequency offset from the central frequency of the transition, corrected for the relative velocity of the cloud $\left(\tilde{\nu}_{0}=23.6945 \mathrm{GHz}-V / c\right.$, with $V_{\mathrm{LSR}}=7.025 \mathrm{~km} \mathrm{~s}^{-1}$ at the peak position). Thin lines: Theoretical spetra for emitting gas with very low opacity $\left(\tau_{\nu} \ll 1 \Rightarrow T_{\mathrm{R}}^{*}(\nu) \propto \tau_{\nu}\right)$. The upper theoretical curve has the same line-width as the displayed measurement. (Here $\Delta V=0.35 \mathrm{~km} \mathrm{~s}^{-1} ; \Delta V$ is the FWHM of the individual Gaussian hyperfine components and is due to thermal, turbulent and instrumental line broadening). The optical depth of the transition can be determined by evaluating the difference between this curve and the measured spectrum in the relative heights of the 5 hyperfine groups. (Here $\tau(1,1, m)=3.3$, where $\tau(1,1, m)$ is the sum of the opacity peaks of the 8 hyperfine components melting together to the main group.) The bottom graph reveals the quantum mechanical transition propabilities of all 18 hyperfine components. A theoretical spectrum with vanishing opacity and line-width would assume this shape.

the outer region). Using this assumption turned out to overestimate $N\left(\mathrm{NH}_{3}\right)$ by at most $60 \%$ and underestimate $N\left(\mathrm{NH}_{3}\right)$ by at most $20 \%$. Hence the detection of the $(J, K)=(1,1)$ main group should be sufficient for a fairly reliable $\mathrm{NH}_{3}$ column density estimate in the outer regions of B217SW. We note that inside the half maximum intensity contour of the $(1,1)$ transition - that is where the most important cloud parameters are to be determined no assumptions regarding $T_{\mathrm{ex}}, T_{12}$ or $\tau$ had to be made.

The size of B217SW is $2.3^{\prime} \times 1.4^{\prime}$, taking the major and minor diameter of the half maximum contour of the $(1,1)$ line area. The geometrical mean diameter is $0.073 \mathrm{pc}=15000 \mathrm{au}$. The column density contours are centred on $\alpha(2000)=4^{\mathrm{h}} 27^{\mathrm{m}} 47^{\mathrm{s}}, \delta=26^{\circ} 18^{\prime} 10^{\prime \prime}$ northeast of the actual peak position with $N\left(\mathrm{NH}_{3}\right)=1.7 \times$ $10^{15} \mathrm{~cm}^{-2}$. As this offset is less than $1 / 2$ of the diagonal step size of the map, it is possible that the offset would get smaller with higher angular resolution observations. Towards the south-east, the peak column density falls of 


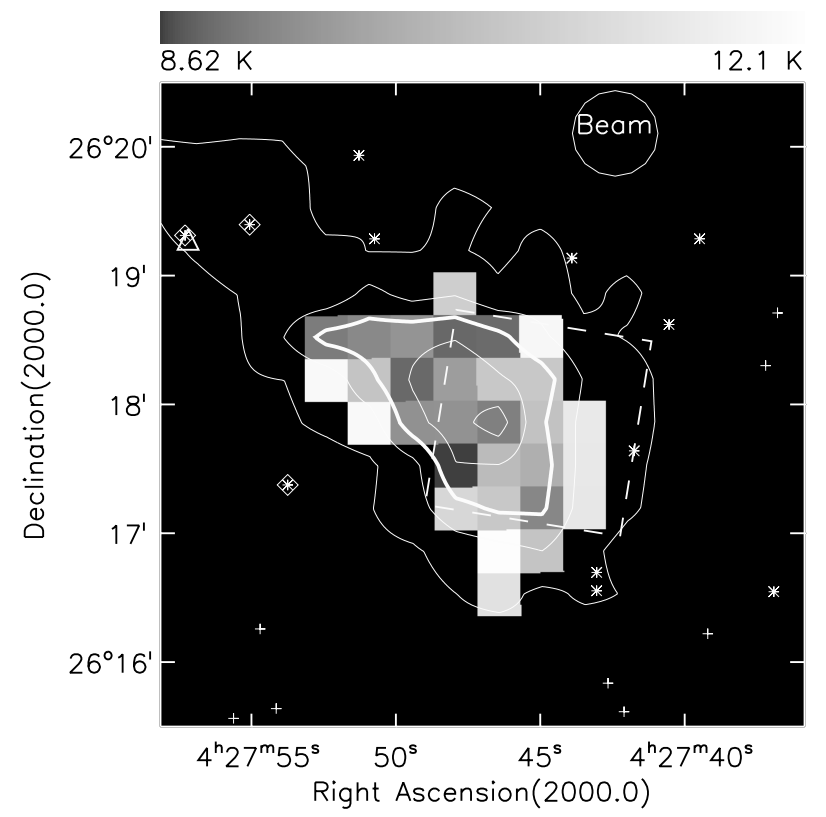

Fig. 3. Kinetic temperature (image) and $\mathrm{NH}_{3}$ column density (contours) of B217SW. $T_{\text {kin }}$ could be determined in the central part only (see text), and its range is indicated by the colour bar at top of the figure. Contours are drawn at 0.1, 0.3, 0.5, 0.7 and $0.9 \times 1.7 \times 10^{15} \mathrm{~cm}^{-2}$, the peak value of $N\left(\mathrm{NH}_{3}\right)$. The half maximum contour is highlighted. The dashed square represents the aperture of one ISOSS detector pixel, and its position and position angle correspond to the scan read-out closest to the ammonia peak (compare Figs. 1 and 9). Plus signs and asterisks mark the positions of 2MASS point sources. Asterisks are used inside the region, where ammonia was detected and $N\left(\mathrm{NH}_{3}\right)$ derived. These objects were used to calculate $\chi\left(\mathrm{NH}_{3}\right)$ except for the possibly embedded stars encased by diamonds (one of which is coincident with IRAS 04248+2612 marked by the triangle). Note that all stars used for the analysis are close to or outside the $10 \%$-contour.

by a factor of 10 in just $1^{\prime}$, while the decline in the other directions is less steep.

The gas temperature is less homogeneous than found in other cores (e.g. by Lemme et al. 1996). The kinetic temperature varies by $3 \mathrm{~K}$ over the densest part of the core, while the typical uncertainty in $T_{\text {kin }}$ is $\sim 1.5 \mathrm{~K}$. Despite this variation, an inside out gradient can be identified, as shown in Fig. 4. The sign of the overall temperature gradient holds to a 3 -sigma level.

The number density of molecular Hydrogen $n\left(\mathrm{H}_{2}\right)$ can be estimated from $\mathrm{NH}_{3}$ data by balancing collisional excitation against emission (Ho \& Townes 1983; we used again the rate coefficients from Danby et al. 1988). This number is close to, but not equal to, the maximum density along the line of sight. The quantities $T_{\mathrm{ex}}$ and $T_{\text {kin }}$ implicitly sample the source function along the line of sight, but are dominated by the region of highest density. The method fails if the density gets high enough to thermalise the $(1,1)$ inversion transition $\left(T_{\mathrm{ex}}=T_{\text {kin }}\right)$, which is the case in the centre of our map. Here we derive a lower limit for the

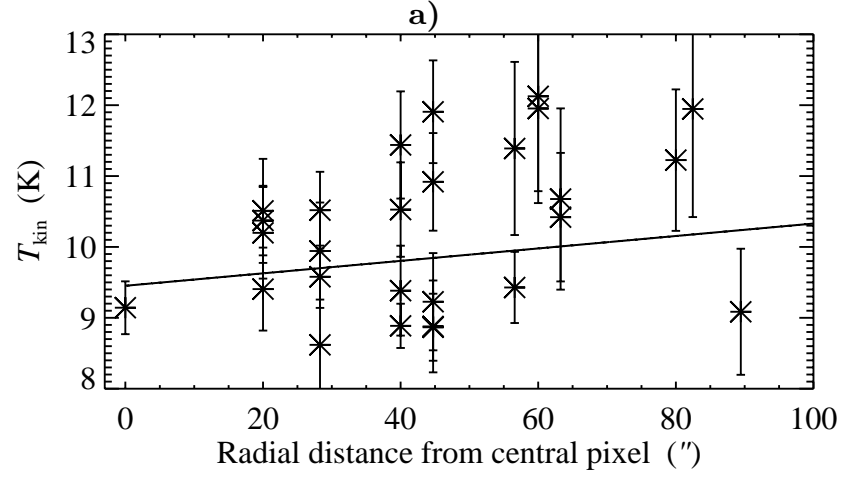

b)

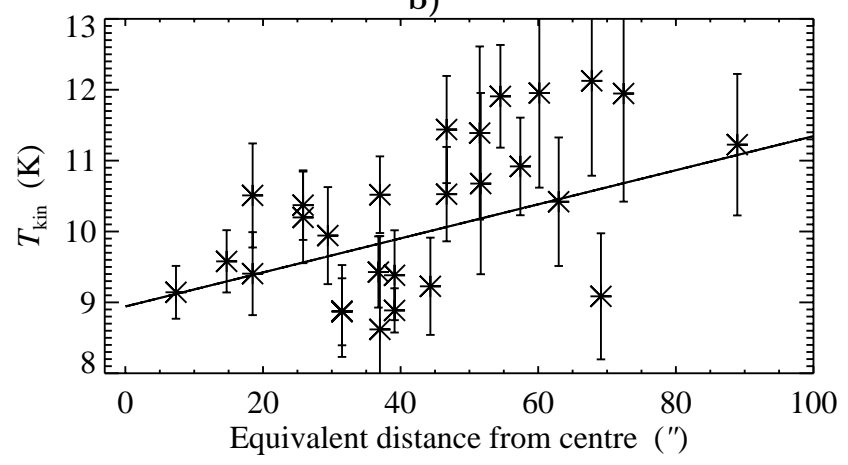

Fig. 4. Radial distribution of the kinetic temperature. Error bars are 1-sigma uncertainties. a) The abscissa values are the radial distances to the peak position in the $\mathrm{NH}_{3}$ column density map (Fig. 1). $\chi^{2}$-fitting of a radial temperature gradient reveales an outward temperature increase of $0.53 \mathrm{~K} /{ }^{\prime}$ (solid line). b) Here, the abscissa values are calculated taking the aspect ratio of the dense core into account: offsets in the direction of the major and minor axes are multiplied by $(2.3 / 1.4)^{-1 / 2}$ and $(2.3 / 1.4)^{1 / 2}$ respectively. before calculating the angular distance. Additionally, it is assumed here that the true centre of the dense core is not in the centre of the map pixel, but shifted by $1 / 3$ of a map pixel (observational step size) to the east and to the north (as suggested by the contours in Fig. 3). The fitted temperature gradient of $(1.44 \pm 0.43) \mathrm{K} /{ }^{\prime}$ corresponds to $3.5 \mathrm{~K} / 0.1 \mathrm{pc}$.

central density by using $T_{\mathrm{ex}}-\sigma\left(T_{\mathrm{ex}}\right)$ and $T_{\text {kin }}+\sigma\left(T_{\mathrm{kin}}\right)$. Figure 5 shows the radial density profile of B217SW.

To calculate the total mass $M_{\mathrm{NH}_{3}}$ of the dense core, the abundance of ammonia relative to the dominating species $\mathrm{H}_{2}$ and $\mathrm{He}$ has to be known. In the following we assume $\chi(\mathrm{He})=0.2$, i.e. $5 \mathrm{H}_{2}$ molecules per $\mathrm{He}$ atom. Various methods were applied in the past to derive the fractional ammonia abundance $\chi\left(\mathrm{NH}_{3}\right) \equiv N\left(\mathrm{NH}_{3}\right) / N\left(\mathrm{H}_{2}\right)$ of dense cores. Benson \& Myers (1983) compared $\mathrm{NH}_{3}$ with $\mathrm{C}^{18} \mathrm{O}$ abundances in 10 nearby dense cores (most of them in Taurus) and found $\chi\left(\mathrm{NH}_{3}\right)$ varying between $3 \times 10^{-8}$ and $2 \times 10^{-7}$, where the $N\left(\mathrm{C}^{18} \mathrm{O}\right)$ to $N\left(\mathrm{H}_{2}\right)$ conversion by Frerking et al. (1982) was used. Recent findings that CO is depleted in cold, dense cores (Willacy et al. 1998; Caselli et al. 1999) suggest that these values might be too high. On the other hand, $\mathrm{C}^{18} \mathrm{O}$ traces also less dense regions than $\mathrm{NH}_{3}$ and these two effects could partially cancel out. Taking the rounded average of this sample yields $\mathrm{NH}_{3}=10^{-7}$. Harju et al. (1993) calculated 
a)

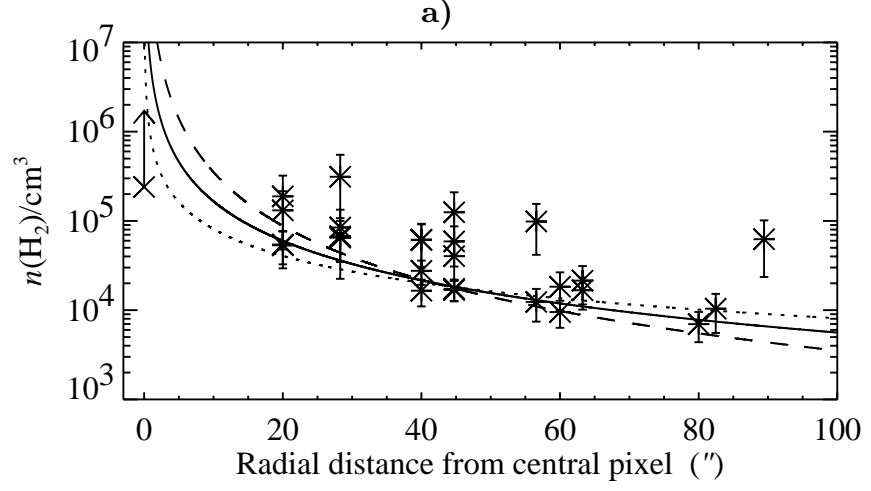

b)

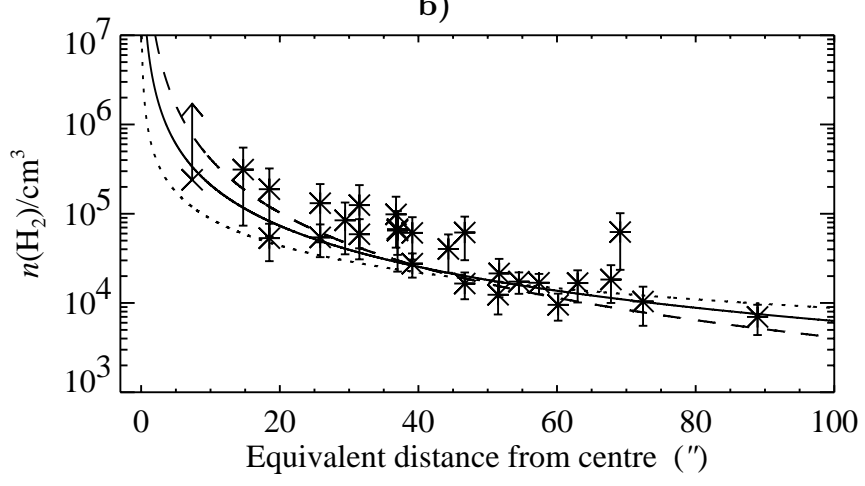

Fig. 5. Radial distribution of the $\mathrm{H}_{2}$ number density with 1-sigma error bars. At the peak position only a lower limit can be given, indicated by an arrow at the leftmost data point (see text for details). The solid lines represent $\chi^{2}$-fits of a power law $\left(n(r) \propto r^{-p}\right)$. For comparison, the dotted and the dashed curves represent $n \propto r^{-1}$ and $n \propto r^{-2}$ density declines respectively. a) and b) differ in the way the angular distance is calculated (see Fig. 4). The best fitting power law exponents are $p=1.5 \pm 0.3$ in both, a) and $\mathbf{b})$.

$\chi\left(\mathrm{NH}_{3}\right)=N\left(\mathrm{NH}_{3}\right) /\left(\operatorname{Ln}\left(\mathrm{H}_{2}\right)\right)$ (where $L$ denotes the assumed core size along the line of sight, taken to be twice the half-power diameter) on the basis of $\mathrm{NH}_{3}$ data alone and found a median of $2.6 \times 10^{-8}$ for 22 ammonia clumps in Orion. They obtained similar results when comparing their $\mathrm{NH}_{3}$ column densities with ${ }^{13} \mathrm{CO}$ data. The difference in the average $\chi\left(\mathrm{NH}_{3}\right)$ values of the Taurus sample (Benson \& Myers 1983) and the Orion sample (Harju et al. 1993) is likely to be a systematic effect of the different methods applied and/or the different regions investigated. However, the scatter in $\chi\left(\mathrm{NH}_{3}\right)$ for the Orion sources as well as for the Taurus sources is large and probably intrinsic to the sources. Therefore we have investigated B217SW itself.

First we have followed Harju et al. (1993) calculating $N\left(\mathrm{H}_{2}\right)=\operatorname{Ln}\left(\mathrm{H}_{2}\right)$. Instead of using this method for statistical analysis in samples of cores we have applied it to all positions in one core and found a median $\chi\left(\mathrm{NH}_{3}\right)=4.4 \times 10^{-8}$. For comparison, we have also derived $N\left(\mathrm{H}_{2}\right)$ performing a NIR reddening analysis using the point source catalogue of the Two Micron All Sky Survey (2MASS, Cutri 1997). There are 12 stars in the region of our map, where $N\left(\mathrm{NH}_{3}\right)$ could be derived (see
Fig. 3). Using the method presented by Wilking et al. (1997), field stars are disentangled from possibly embedded stars (diamonds in Fig. 3) using colour-magnitude $(K$ vs. $H-K)$ and colour-colour $(J-H$ vs. $H-K)$ diagrams. The remaining 9 objects have $J, H$ and $K$ magnitudes compatible with magnitudes of reddened lowmass dwarf or giant stars. Converting $J-K$ colours to $\mathrm{H}_{2}$ column density we follow Lehtinen \& Mattila (1996 and references therein) using $(J-K)_{0}=1.1 \mathrm{mag}$ and Harjunpää \& Mattila (1996 and references therein) using $N\left(\mathrm{H}_{2}\right) / E(J-K)=5.4 \times 10^{-21} \mathrm{~cm}^{-2} / \mathrm{mag}$. Comparing these values with our $\mathrm{NH}_{3}$ column densities we get $\chi\left(\mathrm{NH}_{3}\right)=(2.9 \pm 1.2) \times 10^{-8}$, where the uncertainty is the sample standard deviation. On this basis, we will assume in the following $\chi\left(\mathrm{NH}_{3}\right)=4 \times 10^{-8}$ while regarding $10^{-7}$ and $2.6 \times 10^{-8}$ as upper and lower limit respectively.

For the $2.3^{\prime} \times 1.4^{\prime}$ region inside the half maximum contour we derive a total mass of the core $M_{\mathrm{NH}_{3}}=2.4 M_{\odot}$. Integrating further outwards down to the detection limit we find $M_{\mathrm{NH}_{3}}=4.5 M_{\odot}$. The error is less than $40 \%$ if one neglects the uncertainty in $\chi\left(\mathrm{NH}_{3}\right)$, which is roughly a factor 2 (see above).

To assess the dynamical state of the core, we have compared the gravitational with the kinetic energy, the latter consisting of thermal, turbulent and systematic motions. All of these four energies are calculated on the basis of our $\mathrm{NH}_{3}$ column density map, using only pixels inside the half maximum contour. With assumptions for $\chi(\mathrm{He})$ and $\chi\left(\mathrm{NH}_{3}\right)$ this map is converted into mass $(\mathrm{d} M)$ and total number of particles $(\mathcal{N})$ along each line of sight. Additionally we use maps of $T_{\text {kin }}$ for $E_{\text {therm }}$, $V_{\text {lsr }}$ for $E_{\text {syst }}$ (which includes rotation), and $\Delta V_{\text {turb }} \equiv$ $\left(\Delta V^{2}-\Delta V_{\text {inst }}^{2}-8 \ln 2 k T / 17 m_{\mathrm{H}}\right)^{0.5}$ for $E_{\text {turb }}$, where $17 m_{\mathrm{H}}$ is the mass of the ammonia molecule and $\Delta V_{\text {inst }}$ the instrumental line broadening, for which we take the velocity resolution of the spectrometer $0.15 \mathrm{~km} \mathrm{~s}^{-1}$. For $E_{\text {pot }}$ we must estimate the cloud thickness, which is assumed to be $0.094 \mathrm{pc}$ (the major axis FWHM), over which we evenly distribute the mass along each line of sight.

We find $E_{\text {pot }}=-5.7 \times 10^{35} \mathrm{~J}$. Relative to $\left|E_{\text {pot }}\right|$ the other energy estimates are $E_{\text {therm }}=40 \%, E_{\text {turb }}=9 \%$ and $E_{\text {syst }}=4 \%$, which add up to $53 \%$. We conclude that the core is close to hydrostatic equilibrium, which requires $E_{\text {pot }}=-2 E_{\text {therm. }}$. As a velocity gradient of $3.7 \mathrm{~km} \mathrm{~s}^{-1} \mathrm{pc}^{-1}$ in south-west to north-east direction has been detected, rotation is likely to be present, which accounts for most of the systematic motions: using Eq. (5) of Goodman et al. (1993) to calculate the ratio of rotational $\left(E_{\text {rot }}\right)$ to gravitational energy of a core with density distribution $n \propto r^{-2}$ and given mass, radius and angular velocity (with inclination $i$ ) we find $E_{\text {rot }} /\left|E_{\text {pot }}\right|=0.016 / \sin ^{2} i$ (increasing for a shallower density law). Even though rotation is detected, it is unimportant for the energy balance as found in most of the known ammonia cores (Goodman et al. 1993). The low ratio of turbulent to thermal energy is however more rare and almost exclusively found in Taurus cores. Thermal motions provide most of the pressure balancing gravitation, and turbulence is hardly present. 


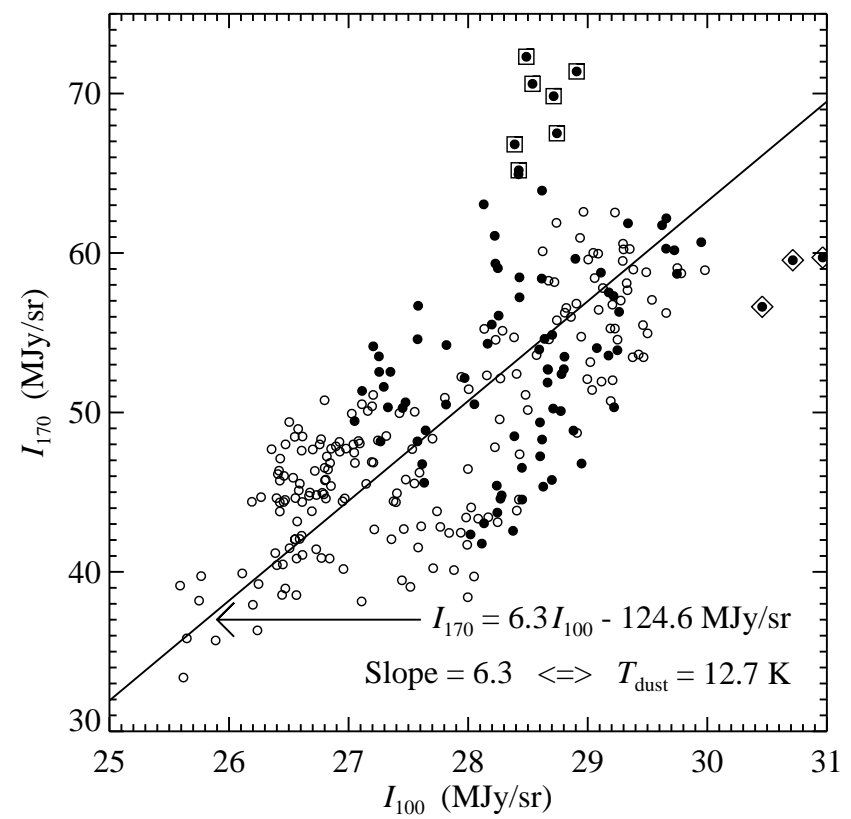

Fig. 6. $I_{170}$ vs. $I_{100}$ correlation plot for B217. All slew data points in the region (as displayed in Fig. 1) are used. As in Fig. 1, filled circles represent read-outs close to the two dense cores, circles encompassed by boxes represent anomalous data points. These show suspiciously high values of $I_{170}$ (upright boxes, " $\square$ ") or $I_{100}$ (diamonds, " $\left.\diamond "\right)$. The solid line is fitted to all the data, its slope corresponds to an average temperature of $12.7 \mathrm{~K}$. Discarding the outliers would change the fit only slightly, the FIR colour temperature would be $12.9 \mathrm{~K}$.

The most crucial parameter assumed for computing the energies is the ammonia abundance $\chi\left(\mathrm{NH}_{3}\right)$, which enters linearly in the kinetic terms but as the square in the gravitational energy. The model of the density distribution along the lines of sight is less crucial, because when calculating potential energies, the spatial distribution in 2 dimensions is taken from the map and only 1 dimension is guessed. Varying the thickness by a factor of 2 changes $E_{\text {pot }}$ less than $25 \%$. Neither of these two ingredients has an impact on the high ratio $E_{\text {therm }} / E_{\text {turb }}=4.4$.

\subsection{Dust}

\subsubsection{Dust temperature and opacity}

The overall dust temperature of B 217 has been derived by fitting a straight line to the $I_{170}-I_{100}$ scatter plot (Fig. 6), using all data points (ISOSS and corresponding HIRES) from the 3 slews in the rectangular region displayed in Fig. 1. The slope corresponds to $T_{\text {dust }}=12.7 \mathrm{~K}$ with a negligible formal error of the line-fit. Taking the uncertainty of the FIR data into account, the result is $T_{\text {dust }}=\left(12.7_{-1.1}^{+2.1}\right) \mathrm{K}$.

The comparison of FIR emission at $100 \mu \mathrm{m}$ and $60 \mu \mathrm{m}$ is shown in Fig. 7. No meaningful average $(100 \mu \mathrm{m} / 60 \mu \mathrm{m})$ colour temperature can be assigned to B217. The lack of correlation between $I_{60}$ and $I_{100}$ can be understood in two ways. Firstly, artefacts due to the nearby FIR point source

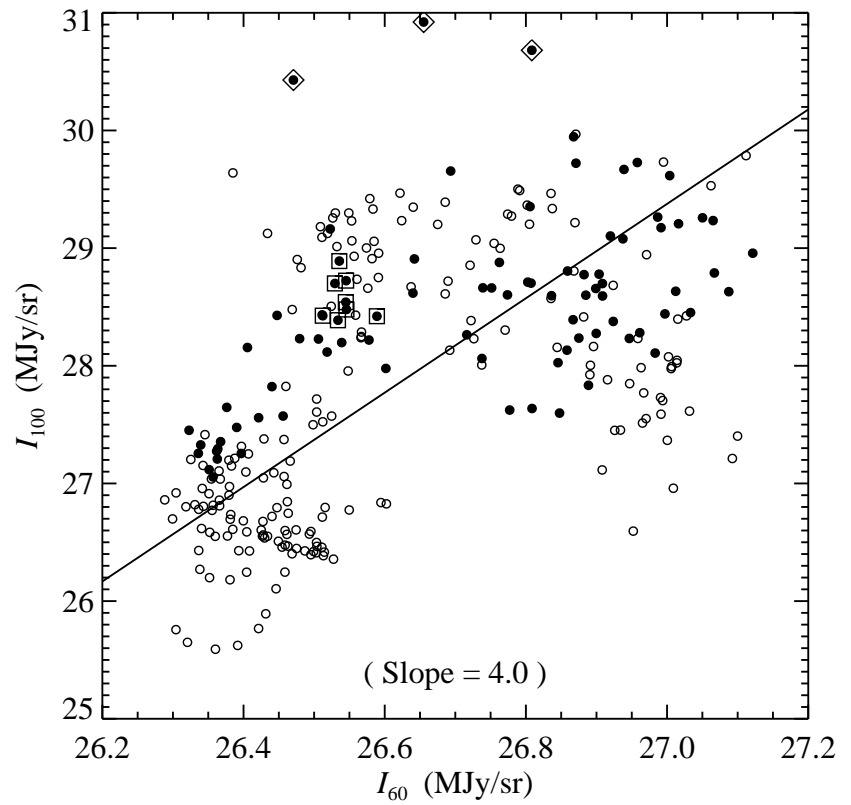

Fig. 7. $I_{100}$ vs. $I_{60}$ correlation plot for B217, using HIRES map read-outs at the same positions as used for Fig. 6. The anomalous data points as defined by Fig. 6 are marked using the same symbols as there. At best some sub-groups of data points show a linear correlation between $100 \mu \mathrm{m}$ and $60 \mu \mathrm{m}$, but colour temperatures derived that way would be arbitrary.

(Sect. 2.1.2) may dominate any real $I_{60}$ emission of B217. Secondly, emission from very small grains (see e.g. Désert et al. 1990) may contribute substantially to $I_{60}$, and this may not be spatially correlated with emission from the big grain component. This latter explanation includes emission from background/foreground structures, which could be stronger than the emission of B217 at $60 \mu \mathrm{m}$ but not at $100 \mu \mathrm{m}$ : if neither big grains of B217 (too cold) nor very small grains of B217 (not present) contribute to $I_{60}$, then one is left with background fluctuations (from standard dust components) and/or map processing artefacts. Dust coagulation in dense cores, which could be responsible for the exceptionally cold big grains (see Sect. 4), would rapidly deplete the very small grains (Ossenkopf 1993 modelling the coagulation process in dense cores with $\left.n>10^{6} \mathrm{~cm}^{-3}\right)$. Observational evidence of this connection is found e.g. by Bernard et al. (1999). Other dark cores without embedded FIR point sources (to exclude artefacts) need to be investigated to confirm the universality of this result.

To estimate the dust temperature of the dense cores themselves we have fitted straight lines to the data points of each of them individually (Fig. 8). For each $\mathrm{NH}_{3}$ core, only read-outs within $5^{\prime}$ radius have been used (cf. Fig. 1). Due to a secondary peak in the FIR data north-west of B217SW (see below) we have excluded slew positions on that side of B217SW. Converting the slopes to colour temperatures we find $T_{\text {dust }}=\left(12.0_{-1.0}^{+1.9}\right) \mathrm{K}$ in B217SW and $T_{\text {dust }}=\left(11.1_{-0.8}^{+1.8}\right) \mathrm{K}$ in B217NE.

Some care must be taken because for a small number of data points an accurate $I_{170}$ vs. $I_{100}$ correlation 


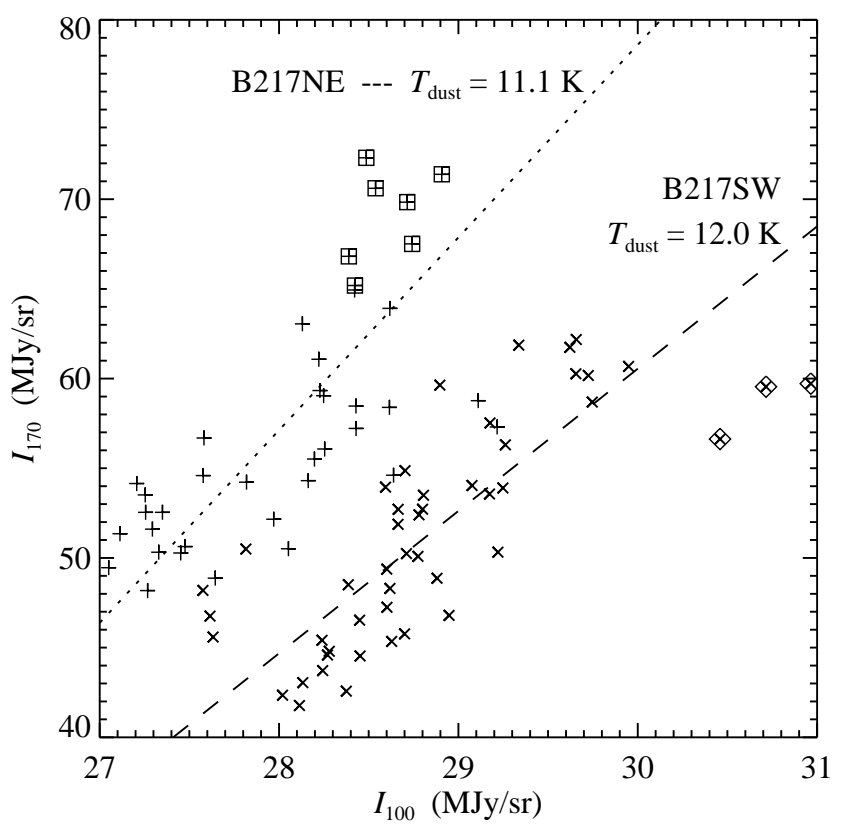

Fig. 8. $I_{170}$ vs. $I_{100}$ correlation plot for the two dense cores B217NE (plus signs, "+") and B217SW (crosses, "×"). Data points used in this figure are marked by filled circles in Figs. 6 and 1. For morphological reasons we used the following selection criteria: Distance $<5^{\prime}$ from the core centres, taking the $I_{170}$ peak position for $\mathrm{B} 217 \mathrm{NE}$ and the $\mathrm{NH}_{3}$ peak position for B217SW, excluding data points beyond the intensity trough (see Fig. 9). Individual fits for B217NE (dotted line) and B217SW (dashed line) correspond to colour temperatures of $11.1 \mathrm{~K}$ and $12.0 \mathrm{~K}$ respectively. Boxes represent anomalous data points as in Fig. 6. With respect to the data of the individual cores however, $I_{170}$-high values do not appear as outliers, but represent the general trend of B217NE. $I_{100}$-high values in contrast, do not match the trend of B217SW. Most likely they are due to artefacts caused by the nearby point source IRAS $04248+2612$ as discussed in Appendix A.2.1. Averaging over a large sample of data points, we can cope with the uncertainty introduced by these artefacts.

is limited by (a) ISOSS flatfielding uncertainties, (b) HIRES processing artefacts due to the nearby IRAS point source. (a) is critical when using individual detector pixels, (b) is critical when using a small region only. For an estimate of the uncertainty in the derived dust temperature of the cores, we assume that structures up to $1 \mathrm{MJy} \mathrm{sr}^{-1}$ in the HIRES map can be introduced by the nearby YSO (Appendix A.2.1). As the $100 \mu \mathrm{m}$ emission of the two cores is of the order $2 \mathrm{MJy} \mathrm{sr}^{-1}$ (offset of $I_{100} \approx 27 \mathrm{MJy} \mathrm{sr}^{-1}$ in Fig. 8 is background) this would distort the linear correlation in Fig. 8, but it cannot be responsible for the good correlation in the first place. Particularly B217SW shows some anomalous data points, which can be explained by this artefact. It is however not justified to exclude the data points, which apparently do not fit the general trend, because all $I_{100}$-values may be affected. Instead, these effects have been taken into account with the error estimate, which is the quadratic sum of the formal line-fit error and the $43 \%$ of the FIR data (see above). In case of B217NE,
$I_{100}$ does not exceed $29 \mathrm{MJysr}^{-1}$, leaving some data points with suspiciously high $I_{170}$ values. The finding that $100 \mu \mathrm{m}$-intensities become saturated for $A_{V} \gtrsim 2 \mathrm{mag}-$ $5 \mathrm{mag}$ was found before in other cores (Laureijs et al. 1994; Boulanger 1994) and is the imprint of a temperature decrease in the inner regions. Consequently the derived average temperature in B217NE is slightly lower than in B217SW. However, the uncertainties discussed above do also apply here (even though artefacts are less apparent), and within the uncertainties the temperatures of B217SW and B217NE are equal. The temperatures derived this way are still averaged over $\sim 50$ data points and therefore not too sensitive to the anomalous data points. But the artefacts on small angular scales discussed above do not allow us to trace temperature differences inside the dense cores.

There is a second $I_{170}$-peak only $3^{\prime}$ northwest of B217SW (see Fig. 9) at $\alpha(2000)=4^{\mathrm{h}} 27^{\mathrm{m}} 33^{\mathrm{s}}, \delta=26^{\circ} 20^{\prime}$. We are lacking any velocity information and cannot discriminate between a separate dense core, detached in space, and a secondary peak of the same core. As the ammonia clump is clearly identified with one of the $I_{170}$ maxima, we regard the secondary peak as independent core and name it B217NW. With a FWHM of $4.4^{\prime}$ in north-south direction it has a similar size as B217SW. The temperature is derived in the same way as for B217SW (not included in Fig. 8 for the sake of visibility), while again no data points on the other side of the intensity valley have been used, thus allocating data points either to B217SW or to B217NW. With $T_{\text {dust }}=\left(13.3_{-1.2}^{+2.4}\right) \mathrm{K}$, $\mathrm{B} 217 \mathrm{NW}$ is slightly warmer than the two dense cores seen in the optical and in the ammonia maps. As the derived "average" temperature of B217 is influenced by B217NE and B217SW, the temperature of B217NW might actually be representative for the average temperature of the lower density regions of B217.

We have calculated the FIR opacity of B217SW from Slew B. After subtracting a linear baseline to exclude FIR background emission (cf. Fig. 9) we find $I_{170, \max }(\mathrm{B} 217 \mathrm{SW})=16 \mathrm{MJy} \mathrm{sr}^{-1}$ and $\tau(170)=0.0025$. Converting this value to hydrogen column density we get $N\left(\mathrm{H}_{2}\right)=N_{\mathrm{H}} / 2=3.6 \times 10^{21} \mathrm{~cm}^{-2}$, where the value for the $170 \mu \mathrm{m}$ absorption cross section per H-nuclei $\left(\sigma_{\mathrm{H}}=\right.$ $\left.3.5 \times 10^{-25} \mathrm{~cm}^{2}\right)$ has been taken from Lehtinen et al. (1998, scaled with factor $(200 / 170)^{2}$ from their $200 \mu \mathrm{m}$ value).

\subsubsection{Flux density and dust mass}

We have used different methods to estimate the total flux densities of the dark core and its dense cores. For B217SW the 1-dimensional scans (Fig. 9) were taken to model a 2dimensional Gaussian. As Slew B is crossing the core parallel to its minor axis, the width in this direction can be accurately measured. Due to the proximity of B217NW, the Gauss function was fitted to the south easterly wing only (right hand side in Fig. 9), and a FWHM of $4.4^{\prime}$ was found. The actual $I_{170}$ peak is measured by Pixel 4 , passing $1.7^{\prime}$ southwest of the $\mathrm{NH}_{3}$ peak. Flatfielding 


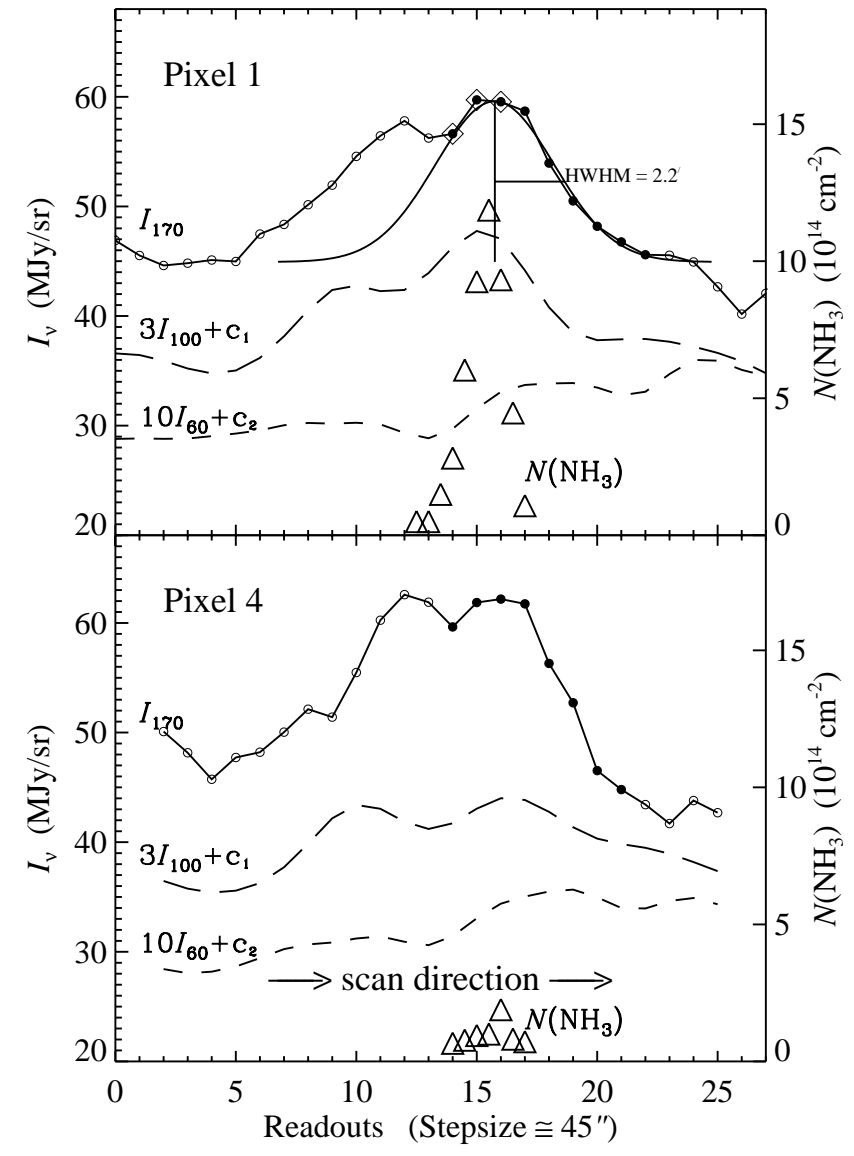

Fig. 9. Pixel 1 (upper plot) and Pixel 4 (lower plot) of Slew B crossing B217SW (see Fig. 1). The slew is crossing the ammonia core perpendicular to its major axis. Pixel 1 is crossing the core almost centrally (see Fig. 3), Pixel 4 runs $75^{\prime \prime}$ off. The plots show the profiles of $I_{170}$ (solid line), $I_{100}$ (long dashes), $I_{60}$ (short dashes) and $\mathrm{NH}_{3}$ column density (triangles). $I_{100}$ and $I_{60}$ are multiplied by 3 and 10 respectively (and shifted by suitable constants $c_{1}=-45 \mathrm{MJy} \mathrm{sr}^{-1}, c_{2}=-235 \mathrm{MJy} \mathrm{sr}^{-1}$ ) for easier comparison with $I_{170}$. Values for $N\left(\mathrm{NH}_{3}\right)$ can be read from the right hand side $y$-axis. Overplotted on the $I_{170}$-curve are the same symbols as used in Figs. 1 and 6 in order to faciliate identification of positions. The Gauss function is used for estimating the FIR flux density (see text). Pixel 4, passing $1.7^{\prime}$ off the $\mathrm{NH}_{3}$ peak position, measures the peak intensity, but confusion with the FIR core northwest of B217SW is likely. An offset between the $\mathrm{NH}_{3}$ peak and the FIR peak of B217SW is however possible, as the dust emission originates in a larger volume than the ammonia emission. See text and Fig. 7 for a discussion of $I_{60}$.

inaccuracies can also lead to $10 \%$ differences between pixels, but most likely the measured $I_{170}$ peak is due to a projection on the north-westerly FIR core. Therefore we have used Pixel 1 for the determination of the source profile. Taking the shape to be circularly symmetric, we get $F_{170} \equiv F_{\nu}(170 \mu \mathrm{m})=27 \mathrm{Jy}$.

In a second method to estimate the flux density of B217SW we have performed aperture photometry. The HIRES map $(100 \mu \mathrm{m})$ cannot be used, because $I_{100}$ is contaminated by the YSO point source over half of the area of $\mathrm{B} 217 \mathrm{SW}$ and the $100 \mu \mathrm{m}$ surface brightness is too low to allow a reliable background subtraction. Therefore a $170 \mu \mathrm{m}$ slew map has been created, which is incomplete, but covers B217SW to $\sim 70 \%$ including the central position. The strong point of the slew map is to take advantage of the 2-dimensional information provided by the different pixels and slews. Taking the background to be the median of the slew map values south of B217SW, and separating emission of B217SW from the secondary peak by cutting along the valley between the two maxima (compare Fig. 9), the integrated intensity sums up to $F_{170}=17 \mathrm{Jy}$. This number underestimates the flux density because of the incomplete coverage at the northeast and the sharp cut at the northwest of the core. Together this may account for the $37 \%$ flux loss compared with the value from the Gaussian fitting. However, these considerations seem to exclude a value of $\sim 50 \mathrm{Jy}$, which would result from the Gaussian method assuming the FIR axis ratio to be the same as in ammonia.

FIR emission of B217NE is more centrally peaked than that of B217SW. The FWHM is $3.4^{\prime}$ in the east-west direction. The relative signals of the four pixels are in agreement with a north-south extension of $3.4^{\prime}$, too. Assuming a circular shape, the flux density is $22 \mathrm{Jy}$. Pixels $2 \& 4$ show the strongest signals, i.e. the peak emission is covered by the slew. The coordinates are $\alpha=4^{\mathrm{h}} 28^{\mathrm{m}} 08^{\mathrm{s}}$, $\delta=26^{\circ} 22^{\prime} 30^{\prime \prime}$, which is $1.5^{\prime}$ north of the brightness minimum in the optical. As there is no second slew crossing, the slew map does not give any additional information. For B217NW we have used the same method as for B217SW as the half width at half maximum (HWHM) is the same in both cores. The derived flux density of B217NW is $32 \mathrm{Jy}$.

The flux density of the whole dark core B217 is difficult to derive, because the profile is not Gaussian and the coverage of the slew map is only $\approx 50 \%$. The integrated flux from the slew map is $F_{170}=95 \mathrm{Jy}$, hence the total flux density regarding the coverage is about $200 \mathrm{Jy}$. This value can be complemented with the integrated flux at $100 \mu \mathrm{m}$, which is $47 \mathrm{Jy}$ in a region of $280 \square^{\prime}$ (slightly larger than the dark field in Fig. 1). Subtracting the 9 Jy point source (attributing it to hot circumstellar dust) and using the derived average temperature of $12.7 \mathrm{~K}$, the remaining $100 \mu \mathrm{m}$ flux density corresponds to $F_{170}=230 \mathrm{Jy}$, which is in reasonable agreement with the slew map value. A factor 2 uncertainty must be assumed due to an uncertain background determination (low $100 \mu \mathrm{m}$ surface brightness of B217 and small scale artefacts from the bright YSO). Taking the rounded average of the two methods, the total flux density of B217 is $F_{170}=(200 \pm 100) \mathrm{Jy}$.

The flux densities can be converted to mass, taking $D$ and $\chi(\mathrm{He})$ as in Sect. 3.1 and using $\sigma_{\mathrm{H}}(170)$ as for the column density. The full formula is

$$
M_{\mathrm{FIR}}=\frac{(1+2 \chi(\mathrm{He})) m_{\mathrm{H}} D^{2}}{\sigma_{\mathrm{H}}(170) K\left(170, T_{\mathrm{d}}\right) B_{170}\left(T_{\mathrm{d}}\right)} F_{170},
$$

where $m_{\mathrm{H}}$ is the atomic hydrogen mass, and $K\left(170, T_{\mathrm{d}}\right)$ is the colour correction factor. The dust parameters in B217 are summarised in Table 1. 
Table 1. Parameters of B217 from FIR observations of the dust.

\begin{tabular}{lllll}
\hline Parameter & B217 & B217SW & B217NE & B217NW \\
\hline Diameter $\left(^{\prime}\right)$ & 15 & 4.4 & 3.4 & 4.4 \\
$T_{\text {dust }}(\mathrm{K})$ & 12.7 & 12.0 & 11.1 & 13.3 \\
$M_{\text {FIR }}{ }^{\star}\left(M_{\odot}\right)$ & 13 & 2.6 & 3.8 & 1.5 \\
\hline
\end{tabular}

* Total mass using $\sigma_{\mathrm{H}, 170}=3.5 \times 10^{-25} \mathrm{~cm}^{2}$.

Table 2. Parameters of B217SW from $\mathrm{NH}_{3} 1.3 \mathrm{~cm}$ radio spectroscopic and FIR continuum measurements.

\begin{tabular}{lll}
\hline Parameter & $\mathrm{NH}_{3}$ inversion lines & FIR continuum \\
\hline Size, not deconv. & $2.3^{\prime} \times 1.4^{\prime}$ & $4.4^{\prime} \times 4.4^{\prime}$ \\
Temperature & $T_{\text {kin }}=(9-12) \mathrm{K}$ & $T_{\mathrm{d}}=12 \mathrm{~K}$ \\
Peak col. dens. & $N\left(\mathrm{H}_{2}\right)=4 \times 10^{22} \mathrm{~cm}^{-2}$ & $N\left(\mathrm{H}_{2}\right)=4 \times 10^{21} \mathrm{~cm}^{-2}$ \\
Peak num. dens. & $n\left(\mathrm{H}_{2}\right)>2.5 \times 10^{5} \mathrm{~cm}^{-3}$ & \\
Total mass & $M=2.4 M_{\odot}^{\star \star}$ & $M=2.6 M_{\odot}$ \\
\hline
\end{tabular}

* Using $\chi\left(\mathrm{NH}_{3}\right)=4 \times 10^{-8}, \sigma_{\mathrm{H}, 170}=3.5 \times 10^{-25} \mathrm{~cm}^{2}$ and $D=140$ pc.

${ }^{\star \star} 4.5 M_{\odot}$ if integrating as well outside the half-max contour.

\subsection{Comparison of gas and dust}

The $\mathrm{NH}_{3}$ core B217SW corresponds to a distinct $170 \mu \mathrm{m}$ dust emission peak, which we have identified with B217SW for the good positional agreement. As outlined below, the difference in the intensity profiles from $\mathrm{NH}_{3}$ and FIR observations meets one's expectations. It shows that the two tracers are most sensitive to different regions in the dense core, but does not conflict the overall identification. The gas and dust parameters of B217SW are summarized in Table 2.

The region of detectable dust emission is larger than the ammonia region. This is partly due to the resolution difference between our radio and FIR data but mainly to a real difference in the distributions. $\mathrm{NH}_{3}$ column densities may vary strongly over relatively small distances due to chemical effects, leading to the well defined entities of dense cores. The FIR continuum on the other hand is more strongly affected by the emission of the host cloud. The bulk of the dust emission comes from a shell around the densest region, because the dust temperature is expected to decrease deep inside the core, while $\mathrm{NH}_{3}$ emission remains detectable throughout the dense core as long as no $\mathrm{NH}_{3}$ freeze out onto grains has set in.

The difference in the intensity profiles of $N\left(\mathrm{NH}_{3}\right)$ and $I_{170}$ as revealed in Fig. 9 is quantified in Table 2 in the order of magnitude difference of equivalent $\mathrm{H}_{2}$ column densities. The effect of the resolution difference can be assessed by averaging $\mathrm{NH}_{3}$ column densities in the aperture of the ISOSS detector pixel covering the ammonia peak position (see Fig. 3). The average column density is a factor of 2.1 lower than its peak value. Apart from this geometrical effect of the pixel's field of view, the flux density of a sharp column density peak as in ammonia would not entirely fall on one detector pixel $(\leq 64 \%$ of the point spread function falls on one pixel; Laureijs 1999). Hence if the dust emission at $170 \mu \mathrm{m}$ was proportional to the ammonia column density, one would expect the ISOSS measured peak value to be a factor of $2-3$ below the peak value from our ammonia map. Therefore, the measured order of magnitude difference in equivalent $\mathrm{H}_{2}$ column densities cannot be attributed solely to observational aspects. The residual factor of 3-4 shows that the above assumption of proportionality between $I_{170}$ and $N\left(\mathrm{NH}_{3}\right)$ does not hold. Many effects such as change of grain properties or $\mathrm{NH}_{3}$ over-abundance in the innermost core region could be responsible, but the most likely reason is a decreasing dust temperature in the core centre: if the dust temperature decreased by $2 \mathrm{~K}$ in the central $1.5^{\prime}$ as compared to the dense core averaged $12 \mathrm{~K}, 170 \mu \mathrm{m}$ emission would drop by a factor of 4 .

Dust and gas temperatures are almost equal in the lower density regions of the ammonia map. This is not self evident, as thermal coupling between gas and dust is not expected for $n \lesssim 10^{4} \mathrm{~cm}^{-3}$ (Krügel \& Walmsley 1984). It is only expected in a $80^{\prime \prime} \times 40^{\prime \prime}$ region around the central position, where densities $>10^{5} \mathrm{~cm}^{-3}$ have been found. The gas temperature shows an inside out gradient over the area of the core with central values around $9 \mathrm{~K}$, so we expect the dust deep inside the core to be at that temperature, too. A putative temperature gradient of the dust in the same region could however not be traced with our $170 \mu \mathrm{m}$ data, as mentioned above. Instead we have found a temperature difference between the dust of the dense cores $(11 \mathrm{~K}-12 \mathrm{~K}$ for B217SW and B217NE) and the outer regions of B217 (13 K-14 K for B217 "average" and the FIR peak $\mathrm{B} 217 \mathrm{NW}$ ), which are only slightly colder than the widespread cold dust component in Taurus. The typical $(170 \mu \mathrm{m} / 100 \mu \mathrm{m})$ colour temperature for the Taurus region is $15.8 \mathrm{~K}(14.2 \mathrm{~K}$ if recalibrating IRAS with the large scale emission gain factor, discussed in Appendix A.2.2), hence the whole star forming region is colder than the overall $17.5 \mathrm{~K}$ cirrus and typical for the $15 \mathrm{~K}$ "colder emission component" (Lagache et al. 1998).

Mass estimates from FIR and $\mathrm{NH}_{3}$ observations are very sensitive to the parameters $T_{\text {dust }}$ and $\chi\left(\mathrm{NH}_{3}\right)$. A typical $15 \%$ uncertainty in $T_{\text {dust }}$ (Sect. 3.2) results in $50 \%$ uncertainty in $M_{\mathrm{FIR}} . M_{\mathrm{NH}_{3}}$ is proportional to $\chi\left(\mathrm{NH}_{3}\right)^{-1}$, which is uncertain to a factor 2 . Within these limits the comparison of $M_{\mathrm{NH}_{3}}$ and $M_{\text {FIR }}$ provides a consistency check of those two parameters. As the dust emission region has about the size of our ammonia map, the mass derived by integrating all map pixels with reliable ammonia detections is appropriate for comparison with $M_{\mathrm{FIR}}$. Comparing these two numbers, we have found $M_{\mathrm{NH}_{3}}$ (whole map) to be $70 \%$ larger than $M_{\mathrm{FIR}}$. This difference is still consistent regarding the accuracy that could be expected. We note that the discrepancy points towards a lower dust temperature and/or a higher fractional ammonia abundance. For example, a dust temperature of $11 \mathrm{~K}$ (lower end of our temperature error bar) would increase $M_{\text {FIR }}$ by $120 \%$.

Finally we have compared the overall dust emission of B217 with the $\mathrm{C}^{18} \mathrm{O}$ survey of Onishi et al. (1998). The $\mathrm{C}^{18} \mathrm{O}$ intensity profile of B217 (core No. 22 in their Fig. 6a) 
has 3 local maxima: one of which at $\alpha(2000)=4^{\mathrm{h}} 28^{\mathrm{m}} 40^{\mathrm{s}}$, $\delta=26^{\circ} 18^{\prime} 30^{\prime \prime}$ is $\sim 2^{\prime}$ south of the path of Slew A. A second $\mathrm{C}^{18} \mathrm{O}$ peak at $\alpha=4^{\mathrm{h}} 28^{\mathrm{m}} 08^{\mathrm{s}}, \delta=26^{\circ} 22^{\prime} 10^{\prime \prime}$ coincides with B217NE. The third $\mathrm{C}^{18} \mathrm{O}$ peak at $\alpha=4^{\mathrm{h}} 27^{\mathrm{m}} 41^{\mathrm{s}}$, $\delta=26^{\circ} 19^{\prime} 50^{\prime \prime}$ is close to B217NW. The total mass of the $\mathrm{C}^{18} \mathrm{O}$ core is $21.4 M_{\odot}$. Seeing that the actual peak position of the $\mathrm{C}^{18} \mathrm{O}$ core is not covered by the ISOSS slews this is in reasonable agreement with $M_{\mathrm{FIR}}=13 M_{\odot}$.

There is no feature in the $\mathrm{C}^{18} \mathrm{O}$ map at the position of the $\mathrm{NH}_{3}$ core. Both $\mathrm{C}^{18} \mathrm{O}$ emission and $170 \mu \mathrm{m}$ dust emission are good tracers of column density in regions with number density $\sim 10^{3}-10^{4} \mathrm{~cm}^{-3}$ (Onishi et al. 1996; Tóth et al. 2000), which is confirmed by the comparison in B217. One would expect both tracers to be sensitive at least to the outer regions of the $\mathrm{NH}_{3}$ core, too. The finding that this is the case for $I_{170}$ but not for $\mathrm{C}^{18} \mathrm{O}$ suggests molecular depletion. We note that due to the large area covered by ISOSS and $\mathrm{C}^{18} \mathrm{O}$ surveys, the $I_{170}-\mathrm{C}^{18} \mathrm{O}$ comparison may provide a useful tool for locating $\mathrm{CO}$ depleted cores.

\section{Discussion}

\subsection{Dynamical and evolutionary state of B217SW}

Our ammonia observations have revealed that B217SW is a strongly centrally condensed core with a half power radius of $0.037 \mathrm{pc}$, total mass $M_{\mathrm{NH}_{3}}=2.4 M_{\odot}$, central density $n_{0}>3 \times 10^{5} \mathrm{~cm}^{-3}$ and a density distribution $n \propto r^{-1.5}$. No significant turbulent or other non-thermal motions are present. The dense core is supported thermally and is close to hydrostatic equilibrium. Gravitional collapse may have started deep inside the core, but cannot be followed with our ammonia observations. Direct observations of infall motions are possible by studying selfabsorption signatures in appropriate molecular line profiles (Zhou et al. 1993), but no such search has been done on B217SW.

The IRAS source in B217 did not prevent the formation of dense cores in its vicinity, even though the projected distance of $0.1 \mathrm{pc}$ is rather small. A simple explanation is given by the observed nebulosity perpendicular to the axis connecting IRAS $04248+2612$ and B217SW (see Sect. 1). If the main disruptive energy is released in a bipolar outflow, the formation of dense cores off-axis can proceed. B217 attains its smallest extent in the south-east to north-west direction close to the YSO. Also, the minor axis of B217SW, its rotational axis and (to $15^{\circ}$ ) the magnetic field lines (Goodman et al. 1992) all lie in this direction. This coincidence suggests either interaction between IRAS $04248+2612$ and the core, or the overall direction of the magnetic field leaves its mark on all substructures of B217. Regarding the quiescence of the dark core (no apparent increase of $T_{\text {dust }}, T_{\text {gas }}$ or turbulence) an interaction seems unlikely, and it is more appropriate to assume that a relatively undisturbed passing of the ambipolar diffusion phase lead to (almost) parallel formation of three dense cores with subsequent collapse of only one of them.
Alignment between core minor axes with magnetic fields does not occur in all molecular cloud cores. Onishi et al. (1996) found $70 \%$ of the $\mathrm{C}^{18} \mathrm{O}$ cores in Taurus to show alignment within $\pm 30^{\circ}$, while Heyer (1988) found no statistically relevant alignment analysing ${ }^{13} \mathrm{CO}$ cores in the same region.

Ammonia is usually considered as a molecule characterizing later stages of chemical evolution. Myers \& Benson (1983) and Suzuki et al. (1992) found $\mathrm{NH}_{3}$ to be more abundant in older cores, where stars have already formed. Also chemical differentiation (as found in many cores e.g. by Zhou et al. 1994) can often be interpreted as a combination of density and age effects (Kuiper et al. 1996; Willacy et al. 1998). The missing peak in $\mathrm{C}^{18} \mathrm{O}$ column density at the position of B217SW (see core No. 22 of Onishi et al. 1996, 1998) suggests depletion of CO in the dense core studied here, too. A high ammonia abundance in B217SW is therefore consistent with an advanced chemical state, which in turn favours a slow dynamical evolution. This is indeed believable, as the core seems to be near hydrostatic equilibrium, and for the geometrical arguments mentioned above.

\subsection{Dust properties}

The dust temperature in B217 is lower than in the majority of dark cores or globules, where temperatures of $13 \mathrm{~K}-15 \mathrm{~K}$ prevail (Lehtinen et al. 1998; Hotzel et al. 2000). Dust temperatures of $11 \mathrm{~K}-14 \mathrm{~K}$ as in B217 are rare. Two dark cores with similar temperatures are B361 (11 K-13 K, Keene et al. 1983) and L183 (12 K-13 K Lehtinen et al. 2000).

Bernard et al. (1992) modelled IR emission of externally heated dust clouds and predict a $(400 \mu \mathrm{m} / 241 \mu \mathrm{m})$ colour temperature of $15.9 \mathrm{~K}$ in a cloud similar in mass and density structure to B217/B217SW (their model with code 324: $n_{\text {central }}=10^{5} \mathrm{~cm}^{-3}, n \propto r^{-2}, A_{V \text {,central }}=10 \mathrm{mag}$, $\left.M=5.99 M_{\odot}\right)$. This is inconsistent with our results. The radiative transfer modelling can be reconciled with our observations, if dust properties in dense and cold environments differ from the adopted (Désert et al. 1990) ISM dust model. Two mechanisms could be involved. Firstly, ice mantles of molecules such as $\mathrm{H}_{2} \mathrm{O}$ and $\mathrm{CO}$ freeze out onto dust grains at densities of a few times $10^{4} \mathrm{~cm}^{-3}$ (van Dishoeck \& Blake 1998), which will change the absorption properties of the grains. Secondly, coagulation of (ice coated) dust grains shifts the size distribution towards larger diameters. The "steady-state" temperature of $0.3 \mu \mathrm{m}$ grains is $\approx 10 \%$ lower than that of comparable $0.1 \mu \mathrm{m}$ grains (Draine \& Lee 1984). The production of "fluffy" agglomerates (Mathis \& Whiffen 1989) may also affect the albedo and the emissivity of the dust. Lehtinen et al. (1998) found the Thumbprint Nebula to be colder than predicted by the numerical calculations and already suggested grain growth to be responsible for the discrepancy. Bernard et al. (1999) discussed low dust temperatures in the cloud MCLD123.5+24.9, showing 
that neither ISRF intensity variations nor extinction could explain the FIR/Sub-mm SED (based on IRAS, ISOPHOT and PRONAOS data) and proposed coagulation of smaller dust particles on large size grains to be responsible.

\section{Conclusions}

B217 is a dark core containing one Class-I YSO and two dense ammonia cores. Our ammonia observations with the Effelsberg $100 \mathrm{~m}$-telescope have revealed the density and temperature distributions of B217SW. The dense core is supported by thermal pressure only and is approximately in hydrostatic equilibrium. Its minor axis, its rotational axis and the magnetic field lines are aligned and perpendicular to the axis connecting B217SW with the YSO and B217NE. This coincidence, the low degree of turbulence and the high abundance of $\mathrm{NH}_{3}$, all point to a slow dynamical evolution, suggesting that the formation of dense cores in B217 has been governed by ambipolar diffusion. The geometrical configuration suggests that the YSO will not prevent the possible collapse of B217SW despite the slow diffusion process.

The advanced evolutionary stage of B217 is further supported by our dust observations with ISOSS. The colour temperatures of $11 \mathrm{~K}-12 \mathrm{~K}$ in the dense cores cannot be modeled with the same dust composition as in diffuse clouds, but can be explained by a change in dust properties due to agglomeration of dust grains. The lack of detectable $60 \mu \mathrm{m}$ emission from very small grains in B217 provides further support for this interpretation. While there is a close correlation between $\mathrm{C}^{18} \mathrm{O}$ emission and $I_{170}$ over much of the $\mathrm{B} 217$ area, a strong $\mathrm{C}^{18} \mathrm{O}$ deficiency relative to $I_{170}$ marks the position of the $\mathrm{NH}_{3}$ core, providing direct evidence for $\mathrm{CO}$ freeze out onto dust grains in B217SW.

Masses derived from the dust and the gas emission agree reasonably well and are consistent with a fractional ammonia abundance of $3-5 \times 10^{-8}$. Temperature gradients are found for both $T_{\text {gas }}$ and $T_{\text {dust }}$, which are not likely to be directly linked. However, the ranges are similar with $T_{\text {gas }}$ between $9 \mathrm{~K}$ and $12 \mathrm{~K}$ inside $\mathrm{B} 217 \mathrm{SW}$, and $T_{\text {dust }}$ between $11 \mathrm{~K}$ and $14 \mathrm{~K}$ as in $\mathrm{B} 217 \mathrm{NE}$ and the lower density regions of $\mathrm{B} 217$ respectively.

We have attempted for the first time to correlate ISOSS $170 \mu \mathrm{m}$ with IRAS/HIRES data. This has proven to be a useful method of studying dust parameters inside a low mass star forming dark core. As $14 \%$ of the sky are covered in both surveys, this may be a useful new tool to characterize the dust in a large number of clouds and cloud cores.

Acknowledgements. We thank the referee for detailed and helpful comments. This project was supported by Deutsches Zentrum für Luft- und Raumfahrt e. V. (DLR) with funds of Bundesministerium für Bildung und Forschung, grant No. 50 QI 98013 , and by the Academy of Finland, grant No. 1011055.

\section{Appendix A: Calibration accuracies of the FIR data}

\section{A.1. ISOSS calibration}

\section{A.1.1. Positional accuracy}

Stickel et al. (2000) investigated the agreement between ISOSS and IRAS positions for a large number of galaxies and found offsets $<45^{\prime \prime}$ for more than $90 \%$ of their sources. In a more recent investigation of the positional accuracy of the ISOSS slews, $2 \%$ of the slews have been identified as having uncertain positional information. In these cases, a delayed finding of the guide star of the subsequent pointed observation was likely to reduce the accuracy of the gyro drift correction, which is necessary to apply to the slew data. Using unflagged slews only, the inspection of 61 detections of Neptune, Uranus and the brighter asteroids yielded no positional offset perpendicular to the slewing direction (which can be measured more accurately), implying an accuracy of $\sim 15^{\prime \prime}$ (Müller et al. 2001). The positional accuracy parallel to the slew is probably worse, because transient effects of the detector play a role. We did not try any correction for this effect because a high in-scan precision (to better than $45^{\prime \prime}$ ) is not crucial for our analysis.

\section{A.1.2. Photometric accuracy}

The photometric accuracy was checked by Tóth et al. (2000) comparing ISOSS results with dedicated pointed raster maps (mode AOT PHT22), which turned out to agree within $\pm 20 \%$ in surface brightness. The uncertainty in the absolute photometry of the mapping mode itself is again $20 \%$ (Klaas et al. 1998). Hence the photometric accuracy of an individual data point is $30 \%$ in case of extended emission.

The uncertainty originates partially in changing detector response, from slew to slew, within the slew and from pixel to pixel. We have estimated these effects by comparing intensities at all positions covered by more than one slew in a $16^{\circ} \times 16^{\circ}$ region centred on $\mathrm{B} 217$. We have calculated the relative brightness deviations of measurement pairs (deviation from their mean) and find a root mean square deviation of only $7.9 \%$. (Measurement pairs come from different slews and have positional offsets $<30^{\prime \prime}$ between individual pixel positions.) The local accuracy of the flatfield has been checked for Slew C (Fig. 1), where every second pixel runs along the same line. The relative brightness deviations of Pixel $1 \& 2$ are $4.5 \%$, of Pixel $3 \& 4$ are $5.8 \%$ (averaged over a scan length of $1^{\circ}$ ).

\section{A.1.3. Comparison with DIRBE}

We will compare our ISOSS $170 \mu \mathrm{m}$ intensities with IRAS $100 \mu \mathrm{m}$ and $60 \mu \mathrm{m}$ data and have therefore checked in a consistent manner the calibration of both ISOSS and IRAS. As a common reference standard, we have used the COBE/DIRBE data base (Hauser et al. 1991). As there 
is no DIRBE $170 \mu \mathrm{m}$ band, we have interpolated from the $100 \mu \mathrm{m}, 140 \mu \mathrm{m}$ and $240 \mu \mathrm{m}$ bands by fitting a modified blackbody spectrum with a $\nu^{2}$ emissivity law. For this, Eq. (1) has been used iteratively, in order to apply the necessary colour corrections. Colour corrections are necessary for all FIR data, in order to convert the quoted intensities (for which an intrinsic spectrum $I_{\nu} \propto \nu^{-1}$ is assumed by convention), to the real values at the reference wavelength. After interpolation, the new DIRBE $170 \mu \mathrm{m}$ intensities have been transformed to the ISOSS filter-band (inverse colour corrections), so that $I_{170}^{\mathrm{DIRBE}}$ and $I_{170}^{\mathrm{ISOSS}}$ can be directly compared.

The result is shown in Fig. A.1a, where the $16^{\circ} \times 16^{\circ}$ Taurus region has been evaluated. All ISOSS values corresponding to the same DIRBE map pixel $\left(\approx 20^{\prime} \times 20^{\prime}\right)$ are averaged. All DIRBE pixels crossed by one or more ISOSS slews are included, i.e. also a lot of uncompletely covered pixels (coverage $<20 \%$ if crossed by 1 slew only). This (and the fact that the DIRBE beam has twice the pixel size) introduces some scatter, but statistically does not affect this analysis. Fitting a straight line to the data points using the ordinary least-squares bisector (OLSB) method (which treates $x$ and $y$ values equivalently, see Isobe et al. 1990) we derive the relation $I_{170}^{\text {ISOSS }}=$ $1.01 I_{170}^{\mathrm{DIRBE}}+0.1 \mathrm{MJy} \mathrm{sr}^{-1}$ with 1 -sigma errors 0.013 (gain) and $0.52 \mathrm{MJy} \mathrm{sr}^{-1}$ (offset).

\section{A.2. IRAS/HIRES calibration}

\section{A.2.1. Photometric accuracy of low surface brightness features}

Photometric calibration of the HIRES maps has been checked for the two FIR point sources in the field (see Fig. A.2). Aperture photometry from the maps yields $5 \%$ and $15 \%$ higher flux densities compared with the IRAS point source catalog (PSC2) for IRAS 04248+2612 and IRAS $04240+2559$ respectively. This small offset can be attributed to background structures, hence HIRES processing did not change the total flux of point sources. However, the "ringing" around point sources (see e.g. Cao et al. 1997) affects our analysis of low surface brightness features near IRAS 04248+2612. Therefore we are limited to features $\gtrsim 1 \mathrm{Jy}\left(\sim 1 \mathrm{MJy} \mathrm{sr}^{-1}\right.$ over $\left.10 \square^{\prime}\right)$, assuming that $10 \%$ of the YSO flux density (9 Jy at $100 \mu \mathrm{m}$ ) is contained in the ring. If the paths of the ISOSS slews cross this ring (worst case) we expect $I_{100}$-artefacts of at most $1 \mathrm{MJy} \mathrm{sr}^{-1}$ for the cores B217SW and B217NE. The contribution of the point source to $I_{100}$ due to the FWHM of $2.2^{\prime}$ is smaller. The closest approach of a scan to the YSO is $2.9^{\prime}$ (Pixel 3, Slew A). A 9 Jy point source at this distance contributes with $0.16 \mathrm{MJy} \mathrm{sr}^{-1}$. If the FWHM of the map was underestimated by $10 \%$, the contribution is $0.30 \mathrm{MJy} \mathrm{sr}^{-1}$. a)

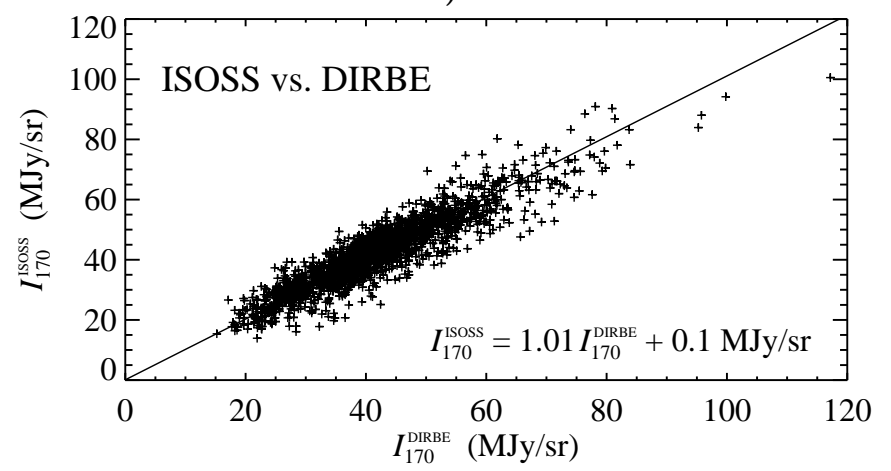

b)

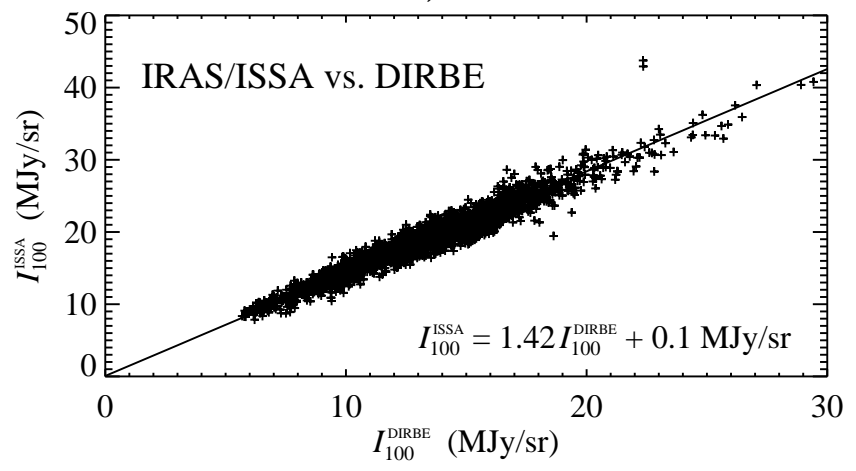

c)

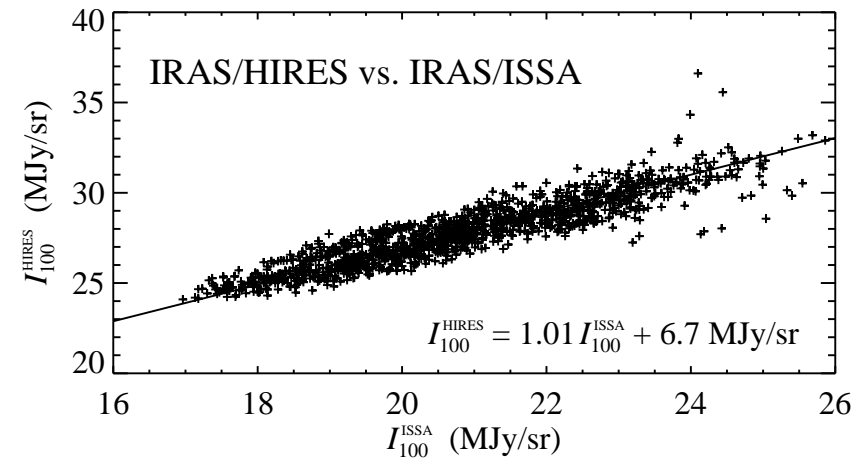

Fig. A.1. Calibration comparison of the FIR data. As the combination of ISOSS with HIRES is a novelty, we check their calibrations against DIRBE. a) ISOSS vs. DIRBE in the $16^{\circ} \times 16^{\circ}$ Taurus region. ISOSS densities are averaged in each DIRBE pixel. b) ISSA vs. DIRBE in the same region as a); again, one data point per DIRBE pixel. c) HIRES vs. ISSA in the B217 area; one data point per ISSA pixel. The maps used are displayed in Fig. A.2. There is a systematic difference between IRAS and DIRBE intensities on these large angular scales, while the calibrations of ISOSS and DIRBE agree. We do not rescale the HIRES data used in this paper, because the gain value for very small angular scales could be different. However, as our investigated structures of sizes $2^{\prime}-15^{\prime}$ clearly exceed the size of point sources (for the IRAS detector), the derived colour temperatures may be systematically too high $\left(I_{170} / I_{100}\right.$ too low).

\section{A.2.2. IRAS/HIRES versus DIRBE}

As we have done with the ISOSS data, we have checked the IRAS calibration against DIRBE. The HIRES maps 


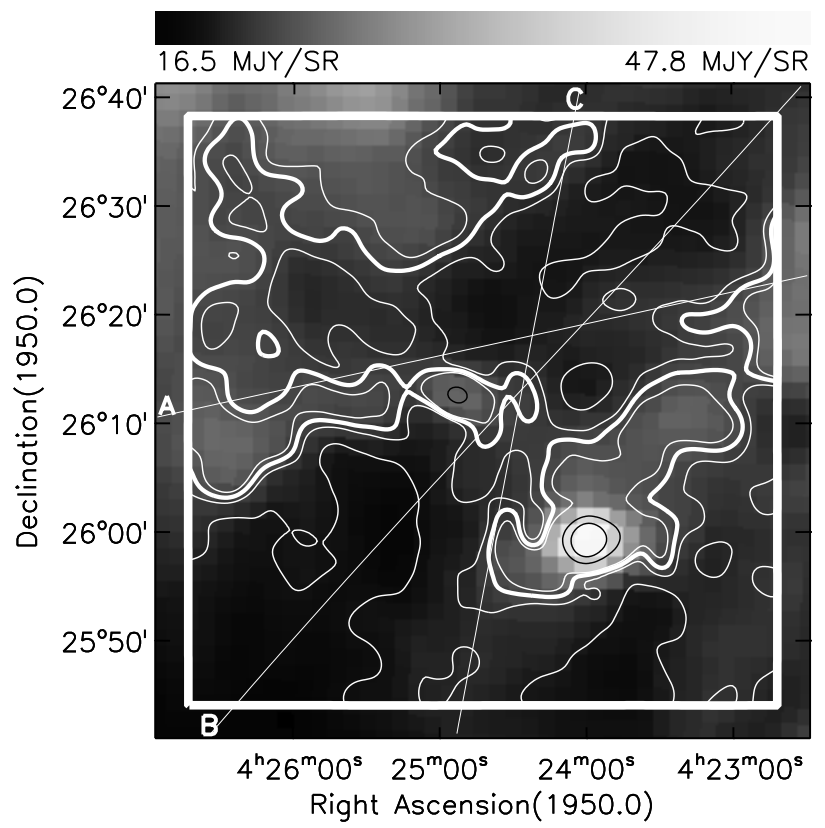

Fig. A.2. The HIRES $100 \mu \mathrm{m}$ map (contours) used for comparison with ISOSS (i.e. smoothed to $2.2^{\prime}$ ) overlayed on the ISSA $100 \mu \mathrm{m}$ map (image), which has been used to check the HIRES calibration in Fig. A.1c. The grey-scale used is indicated at the top of the figure; contours are drawn at 26, 28, 29(thick), 30,40 (black) and 60 (black) $\mathrm{MJy} \mathrm{sr}^{-1}$. The white contours are chosen in order to identify the structure seen in Fig. 9, the black contours are chosen in order to mark the two bright point sources in the field. The fainter one is IRAS 04248+2612 in the centre of B217. The paths of the 3 slews crossing the dark core are overlayed (labeled "A", "B", "C").

are too small to be directly compared, therefore we have used a 2-step procedure comparing first DIRBE with the IRAS/ISSA maps (Wheelock et al. 1994) and subsequently IRAS/ISSA with IRAS/HIRES. The former step is performed in the same $16^{\circ} \times 16^{\circ}$ region used above for the ISOSS-DIRBE comparison, and the result is shown in Fig. A.1b. All ISSA pixels falling in the same DIRBE pixel are averaged. The OLSB fit yields $I_{100}^{\text {ISSA }}=$ $1.42 I_{100}^{\mathrm{DIRBE}}+0.1 \mathrm{MJy} \mathrm{sr}^{-1}$ with 1 -sigma errors 0.012 (gain) and $0.15 \mathrm{MJy} \mathrm{sr}^{-1}$ (offset). This ISSA-DIRBE relation in Taurus is equal (within 1-sigma) to the all-sky relation found by Wheelock et al. (1994). Performing the same comparison for the $60 \mu \mathrm{m}$ data we have found $I_{60}^{\mathrm{ISSA}}=$ $1.34 I_{60}^{\text {DIRBE }}-1.7 \mathrm{MJy} \mathrm{sr}^{-1}$, while the gain derived by Wheelock et al. (1994) is $1.15 \pm 0.07$. It is not surprising that the gain between the $60 \mu \mathrm{m}$ data sets is less homogenous across the sky than the gain between the $100 \mu \mathrm{m}$ data sets, because heterogenous dust populations and environments contribute to $I_{60}$ (big grains/very small grains, interstellar/circumstellar/interplanetary dust), while $I_{100}$ is dominated by big grains of the interstellar dust. For example, the Taurus region is close to the ecliptic, and the zodiacal light was subtracted using diffent models for IRAS and DIRBE.

The HIRES-ISSA comparison is shown in Fig. A.1c for the $1 \square^{\circ}$ region of the HIRES map (shown in Fig. A.2).
The HIRES pixels falling in the same ISSA pixel are averaged, but no further convolution has been performed, hence the two bright point sources in the field introduce some artificial structure. Ignoring the brightest $2 \%$ of the data points, the gain is unity, as expected. For our calibration purposes we can ignore the offset, which is due to zodiacal light that has been subtracted from the ISSA but not from the raw scan data used for the HIRES maps.

Considering the findings of Fig. A.1, $I_{100}$ HIRES values from extended objects must be devided by $1.42\left(I_{60}\right.$ by 1.34) in order to be consistent with the ISOSS calibration. This correction must however not be applied to point sources, as emphasized by Wheelock et al. (1994, p. IV16). For angular scales of the order $2^{\prime}-15^{\prime}$, the correction factor is not known and may even change between its extremes 1 (no correction for structures as the dense cores) and 1.4 (for B217 as a whole). For this difficulty, we have not applied any correction to the HIRES data, bearing in mind that the $I_{170} / I_{100}$ ratios needed for deriving colour temperatures could be systematically too low.

\section{References}

Aumann, H. H., Fowler, J. W., \& Melnyk, M. 1990, AJ, 99, 1674

Benson, P. J., \& Myers, P. C. 1983, ApJ, 270, 589

Bernard, J. P., Boulanger, F., Désert, F. X., et al. 1992, A\&A, 263, 258

Bernard, J. P., Abergel, A., Ristorcelli, I., et al. 1999, A\&A, 347,640

Bogun, S., Lemke, D., Klaas, U., et al. 1996, A\&A, 315, L71

Boulanger, F. 1994, Dust and Gas in the Infrared Cirrus, in The First Symposium on the Infrared Cirrus and Diffuse Interstellar Clouds, ed. R. M. Cutri, \& W. B. Latter, ASP Conf. Ser., 58, 101

Butner, H. M., Lada, E. A., \& Loren, R. B. 1995, ApJ, 448, 207

Cao, Y., Terebey, S., Prince, T. A., et al. 1997, ApJS, 111, 387

Caselli, P., Walmsley, C. M., Tafalla, M., et al. 1999, ApJ, 523, L165

Chen, H., Myers, P. C., Ladd, E. F., et al. 1995, ApJ, 445, 377

Cutri, R. M. 1997, The 2MASS Point Source Processing Pipeline, Design and Prototype Results, in The Impact of Large Scale Near-IR Sky Surveys, ed. F. Garzon, N. Epchtein, \& A. Omont, ASSL, 210, 187

Danby, G., Flower, D. R., Valiron, P., et al. 1988, MNRAS, 235,229

Désert, F. X., Boulanger, F., \& Puget, J. L. 1990, A\&A, 237, 215

Draine, B. T., \& Lee, H. M. 1984, ApJ, 285, 89

Elias, J. H. 1978, ApJ, 224, 857

Frerking, M. A., Langer, W. D., \& Wilson, R. W. 1982, ApJ, 262,590

Fuller, G. A., \& Myers, P. C. 1993, ApJ, 418, 273

Gaida, M., Ungerechts, H., \& Winnewisser, G. 1984, A\&A, 137, 17

Goodman, A. A., Jones, T. J., Lada, E. A., et al. 1992, ApJ, 399, 108

Goodman, A. A., Benson, P. J., Fuller, G. A., et al. 1993, ApJ, 406, 528 
Harju, J. 1989, A\&A, 219, 293

Harju, J., Walmsley, C. M., \& Wouterloot, J. G. A. 1993, A\&AS, 98, 51

Harjunpää, P., \& Mattila, K. 1996, A\&A, 305, 920

Hauser, M. G., Kelsall, T., Moseley, S. H. J., et al. 1991, The Diffuse Infrared Background - COBE and Other Observations, in After the first three minutes, ed. S. S. Holt, C. L. Bennett, \& V. Trimble, AIP Conf. Proc. 222, 161

Heyer, M. H. 1988, ApJ, 324, 311

Ho, P. T. P., \& Townes, C. H. 1983, ARA\&A, 21, 239

Hotzel, S. 2001, Die 170- $\mu$ m-Zufallsdurchmusterung mit ISO Strukturen im kalten Staub der Milchstraße, Ph.D. Thesis, Ruprecht-Karls-Universität Heidelberg, in preparation

Hotzel, S., Lemke, D., Krause, O., et al. 2000, Chamaeleon's Cold Cloud Cores, in Proc. Workshop: ISO Surveys of a Dusty Universe, ed. D. Lemke, M. Stickel, \& K. Wilke (Springer), 259

Isobe, T., Feigelson, E. D., Akritas, M. G., et al. 1990, ApJ, 364,104

Keene, J., Davidson, J. A., Harper, D. A., et al. 1983, ApJ, $274, \mathrm{~L} 43$

Klaas, U., Laureijs, R. J., Radovich, M., et al. 1998, ISOPHOT Calibration Accuracies, SAI/98-092/Dc Version 2, ESA

Krügel, E., \& Walmsley, C. M. 1984, A\&A, 130, 5

Kuiper, T. B. H., Langer, W. D., \& Velusamy, T. 1996, ApJ, 468,761

Lada, C. J. 1987, Star formation - From OB associations to protostars, in Star Forming Regions, ed. M. Peimpert, \& J. Jugaku, IAU Symp., 115, 1

Lada, C. J., \& Wilking, B. A. 1984, ApJ, 287, 610

Lagache, G., Abergel, A., Boulanger, F., et al. 1998, A\&A, 333, 709

Laureijs, R. J. 1999, Point spread function fractions related to the ISOPHOT C100 and C200 arrays, Tech. Rep. Version 1.0, ISO Data Centre, ESA

Laureijs, R. J., Helou, G., \& Clark, F. O. 1994, The 100 Micron Emission of Clouds, in The First Symposium on the Infrared Cirrus and Diffuse Interstellar Clouds, ed. R. M. Cutri, \& W. B. Latter, ASP Conf. Ser., 58, 133

Lee, C. W., \& Myers, P. C. 1999, ApJS, 123, 233

Lehtinen, K., \& Mattila, K. 1996, A\&A, 309, 570
Lehtinen, K., Lemke, D., Mattila, K., et al. 1998, A\&A, 333, 702

Lehtinen, K., Mattila, K., Lemke, D., et al. 2000, ISOPHOT Far-Infrared Survey of Nearby Molecular Clouds, in Proc. Workshop: ISO Surveys of a Dusty Universe, ed. D. Lemke, M. Stickel, \& K. Wilke (Springer), 317

Lemke, D., Klaas, U., Abolins, J., et al. 1996, A\&A, 315, L64

Lemme, C., Wilson, T. L., Tieftrunk, A. R., et al. 1996, A\&A, 312,585

Mathis, J. S., \& Whiffen, G. 1989, ApJ, 341, 808

Mizuno, A., Onishi, T., Yonekura, Y., et al. 1995, ApJ, 445, L161

Müller, T. G., Hotzel, S., \& Stickel, M. 2001, A\&A, in preparation

Myers, P. C., \& Benson, P. J. 1983, ApJ, 266, 309

Myers, P. C., Linke, R. A., \& Benson, P. J. 1983, ApJ, 264, 517

Onishi, T., Mizuno, A., Kawamura, A., et al. 1996, ApJ, 465, 815

Onishi, T., Mizuno, A., Kawamura, A., et al. 1998, ApJ, 502, 296

Ossenkopf, V. 1993, A\&A, 280, 617

Ott, M., Witzel, A., Quirrenbach, A., et al. 1994, A\&A, 284, 331

Stickel, M., Lemke, D., Klaas, U., et al. 2000, A\&A, 359, 865

Suzuki, H., Yamamoto, S., Ohishi, M., et al. 1992, ApJ, 392, 551

Tamura, M., Gatley, I., Waller, W., et al. 1991, ApJ, 374, L25

Tóth, L. V., Hotzel, S., Krause, O., et al. 2000, A\&A, 364, 769 van Dishoeck, E. F., \& Blake, G. A. 1998, ARA\&A, 36, 317

Walmsley, C. M., \& Ungerechts, H. 1983, A\&A, 122, 164

Wheelock, S. L., Gautier, T. N., Chillemi, J., et al. 1994, IRAS sky survey atlas: Explanatory supplement, JPL Publikation 94-11, IPAC, JPL

Wilking, B. A., McCaughrean, M. J., Burton, M. G., et al. 1997, AJ, 114, 2029

Willacy, K., Langer, W. D., \& Velusamy, T. 1998, ApJ, 507, L171

Zhou, S., Wu, Y., Evans, N. J., et al. 1989, ApJ, 346, 168

Zhou, S., Evans, N. J., Koempe, C., et al. 1993, ApJ, 404, 232

Zhou, S., Evans, N. J., Wang, Y., et al. 1994, ApJ, 433, 131 